\title{
Long time existence for the semi-linear beam equation on irrational tori of dimension two
}

\author{
Rafik Imekraz \\ Institut DE MAThÉMATiques DE BordeAux, \\ UMR 5251 DU CNRS, UNIVERSitÉ DE BordeAux, \\ 351, cours de la Libération F33405 Talence Cedex, France
}

May 30, 2016

\begin{abstract}
We prove a long time existence result for the semi-linear beam equation with small and smooth initial data. We use a regularizing effect of the structure of beam equations and a very weak separation property of the spectrum of an irrational torus under a Diophantine assumption on the radius. Our approach is inspired from a paper by Zhang about the Klein-Gordon equation with a quadratic potential.
\end{abstract}

2000 Mathematics Subject Classification : $\quad 37 \mathrm{~K} 45,35 \mathrm{Q} 55,35 \mathrm{~B} 34,35 \mathrm{~B} 35$

Key words : beam equation, Klein-Gordon equation, normal form, irrational torus

\section{Introduction}

In this paper we study the solutions of the so-called beam equation with periodic boundary conditions and with a positive and constant potential :

$$
\left(\partial_{t}^{2}+\Delta^{2}+m^{2}\right) w=w^{n+1}, \quad(x, y) \in[0,2 \pi] \times[0,2 \pi r], \quad t \in \mathbb{R} .
$$

Here, the length $r$ is a positive number, $n \geq 2$ is an integer and $m$ is a positive number. From a Riemannian point of view, the previous setting is equivalent to pose the equation on the compact manifold $\mathbb{S}^{1} \times r \mathbb{S}^{1}$ so $r$ can be interpreted as a radius. Originally, the beam equation has a physical meaning in dimension 1 because it arises in modeling the oscillations of a uniform beam. In dimension 2 , similar equations can be used to model the motion of a clamped plate (see several references in the introduction of [22]). Recent mathematical results have been proven in KAM or scattering frameworks, we refer for instance to the works [15, 16, 21, 23.

Since $\Delta^{2}$ is a fourth-order operator, a natural functional space for the equation (1) is

$$
\mathcal{C}^{0}\left((-T, T), H^{s+2}\left(\mathbb{S}^{1} \times r \mathbb{S}^{1}\right)\right) \cap \mathcal{C}^{1}\left((-T, T), H^{s}\left(\mathbb{S}^{1} \times r \mathbb{S}^{1}\right)\right), \quad T>0, \quad s \in \mathbb{R} .
$$

Using the fact that $H^{s}\left(\mathbb{S}^{1} \times r \mathbb{S}^{1}\right)$ is an algebra for any real number $s>1$ and reformulating the beam equation (1) with the Duhamel formula, one can check that, for any initial data $(w(0), \dot{w}(0))=\left(\varepsilon w_{0}, \varepsilon w_{1}\right)$ in $H^{s+2}\left(\mathbb{S}^{1} \times r \mathbb{S}^{1}\right) \times H^{s}\left(\mathbb{S}^{1} \times r \mathbb{S}^{1}\right)$, if $\varepsilon \in(0,1)$ is small enough then the beam equation (1) admits a unique solution $w$ on a time interval of length $T \gtrsim \varepsilon^{-n}$ such that

$$
\forall t \in(-T, T) \quad\|w(t)\|_{H^{s+2}\left(\mathbb{S}^{1} \times r \mathbb{S}^{1}\right)}+\|\dot{w}(t)\|_{H^{s}\left(\mathbb{S}^{1} \times r \mathbb{S}^{1}\right)} \leq C \varepsilon .
$$

The time $\varepsilon^{-n}$ is called the local existence time. The goal of this paper is to improve the local existence time $\varepsilon^{-n}$ to $\varepsilon^{-A n}$ for some universal constant $A>1$. We will use methods which have been developed in the framework of the semi-linear Klein-Gordon equation on a compact Riemannian manifold $X$ :

$$
\left(\partial_{t}^{2}-\Delta+m^{2}\right) w=w^{n+1}, \quad x \in X, \quad t \in \mathbb{R} .
$$


More precisely, we will adapt the paper [26] to our framework (the differences are explained below). Let us briefly recall what is known about the improvement of the local existence time $\varepsilon^{-n}$ for the equation (2). Using a normal form procedure for a generic parameter $m>0$ and for high regularities $s \gg 1$, one can divide this analysis in two categories :

- if the spectrum of $\sqrt{-\Delta}$ is separated. That means that the difference of two successive eigenvalues of $\sqrt{-\Delta}$ is uniformly bounded from below. For those manifolds, we can improve the local existence time $\varepsilon^{-n}$ to $c(A) \varepsilon^{-A n}$ for any $A>1$ and we usually say that an almost global existence holds for the Klein-Gordon equation (2). The simplest manifold we have in mind is the one-dimensional torus $\mathbb{T}(6,1])$. The case of the sphere $\mathbb{S}^{d}$ indeed appears as a particular case of Zoll manifolds. Eigenvalues of those manifolds have a property of separation which is weaker than the one above. Nevertheless, Bambusi, Delort, Grébert and Szeftel succeeded in proving the almost global existence for the equation (2) on a Zoll manifold (see the paper [3]). Their proof relies on universal multilinear estimates of eigenfunctions proven by Delort and Szeftel in the paper [10]. We also refer to the papers [17, 2, 4, 9, 19, 8, for more on the subject.

- if the spectrum of $\sqrt{-\Delta}$ is not separated. In this category, we have to think to the multidimensional torus $\mathbb{T}^{d}$, for instance if $d \geq 4$ then the eigenvalues of $\sqrt{-\Delta}$ are exactly the numbers $\sqrt{k}$ with $k \in \mathbb{N}$ (so that we have $\lim _{k \rightarrow+\infty} \sqrt{k+1}-\sqrt{k}=0$ ). The multidimensional torus has been studied by Delort in 7 with a new approach of small divisors and he improved the local existence time from $\varepsilon^{-n}$ to $\varepsilon^{-n\left(1+\frac{2}{d}\right)}$ (up to a logarithmic term). This time has been improved to $\varepsilon^{-\frac{3}{2} n}$ by Fang and Zhang (see [11) if $d$ is larger than 4 . In the last two papers, the harmonic analysis of $\mathbb{T}^{d}$ is used, namely the fact that the eigenfunctions of $\mathbb{T}^{d}$ are naturally parametrized by $\mathbb{Z}^{d}$. Later in [26], Zhang proved that the harmonic analysis is not really necessary to get a long time existence by dealing with the harmonic oscillator (the price to pay is to have a weaker improvement of the local existence time equal to $\varepsilon^{-\frac{4}{3} n}$ ). The author generalized the previous paper for superquadratic oscillators in 20 for which we can reach the time $\varepsilon^{-2 n}$ due to a stronger separation of the eigenvalues.

We also refer to the papers [12, 14, 13, 5] for analogue questions on Schrödinger equations and references therein.

Therefore, it is a natural question to study the semi-linear Klein-Gordon equation (2) on a compact manifold with very badly separated eigenvalues. Unfortunately, as in lots of other contexts in partial differential equations, it seems that we need a knowledge about the behavior of the eigenvalues. In contrast, working on the beam equation (1) is easier because it admits a slight regularizing effect due to the operator $\Delta^{2}$ of order 4 . The irrational torus $\mathbb{S}^{1} \times r \mathbb{S}^{1}$ seems to be a good candidate for at least two reasons :

i) it is an explicit example of manifold with a very bad spectral behavior (accumulation of eigenvalues),

ii) if one comes back to the interpretation of $\mathbb{S}^{1} \times r \mathbb{S}^{1}$ as periodic boundary conditions on $[0,2 \pi] \times[0,2 \pi r]$, then one may say that the generic condition is the irrational case $r^{-2} \in \mathbb{Q}$. In other words, irrational tori happens more often than rational tori.

Roughly speaking, our contribution is an adaptation of the method of 26] to get a long time existence result for (1) on an irrational torus $\mathbb{S}^{1} \times r \mathbb{S}^{1}$, we use the property that the very bad separation of the spectrum is counterbalanced by a natural regularizing effect of the beam equation.

From now, we go into details of the description of our main result (Theorem 1.2 below) and we explain the differences with previous works. The first thing to do is looking at the spectrum of the Laplace-Beltrami operator of the torus $X=\mathbb{S}^{1} \times r \mathbb{S}^{1}$ :

$$
\operatorname{Sp}(-\Delta):=\left\{p^{2}+r^{-2} q^{2},(p, q) \in \mathbb{Z}^{2}\right\} .
$$

We expect that the case $r^{-2} \in \mathbb{Q}$ leads to an almost global existence result for the beam equation (this can probably be proven by using methods in the category of manifolds with a separated spectrum, see above). In our paper, we consider the drastically different case where $r^{-2}$ is irrational. Hence, 
multiplicities of eigenvalues are equal to 1,2 or 4 . Let us sort the spectrum of $\sqrt{1-\Delta}$ as an increasing and positive sequence $\left(\lambda_{k}\right)_{k \geq 1}$ without counting multiplicities :

$$
\operatorname{Sp}(I-\Delta)=\left\{\lambda_{1}^{2}<\lambda_{2}^{2}<\lambda_{3}^{3}<\ldots\right\}
$$

From the Weyl law or by a direct computation, one checks that $\lambda_{k}$ is asymptotically equivalent to $\frac{2}{\sqrt{r \pi}} \sqrt{k}$ as $k$ tends to infinity. Such an asymptotic equivalent is simple but not sufficient to write a normal form procedure. We indeed need to understand the behavior of differences of two eigenvalues, or equivalently to get suitable approximations of the number $r^{-2}$ by the rational numbers. As often in dynamical systems, Diophantine conditions naturally appear.

Definition 1.1. An irrational real number $R$ is Diophantine (and we write $R \in \mathcal{D}$ ) if the following holds

$$
\forall \mu>2 \quad \exists C(R, \mu)>0 \quad \forall \frac{P}{Q} \in \mathbb{Q} \quad\left|R-\frac{P}{Q}\right| \geq \frac{C(R, \mu)}{|Q|^{\mu}} .
$$

The condition $\mu>2$ in (3) will play a role in the improvement of the local existence time of the beam equation. Let us recall that in the previous definition, we cannot expect that $\mu$ is less than 2 in (3) for an irrational number $R>0$ since Dirichlet's approximation theorem states that there are infinitely many rationals numbers $\frac{P}{Q}$ such that

$$
\left|R-\frac{P}{Q}\right|<\frac{1}{Q^{2}}
$$

The study of irrationality measures of real numbers has a long history and we only state the results which prove that the set $\mathcal{D}$ of the Diophantine numbers is not empty :

a) almost any real number $R$ in the sense of Lebesgue belongs to $\mathcal{D}$ (this is a classical fact and it is easy to prove by using the convergence of the series $\sum Q^{-(\mu-1)}$ with $Q$ running over $\mathbb{N}^{\star}$ and $\mu \in(2,+\infty)$ is fixed),

b) there are also numbers $R$ such that one can choose $\mu=2$ in (3). Such numbers are called "badly approximable", for instance irrational quadratic numbers are convenient,

c) any irrational algebraic number belongs to $\mathcal{D}$. For instance, $\sqrt[d]{2}$ belongs to $\mathcal{D}$ for any integer $d \geq 2$. This is the famous Roth theorem [24].

We refer for instance to [18, Part D] or [25, Chapter II] for more about the theory of Diophantine approximations. We can now state our main result about the beam equation (1).

Theorem 1.2. Assume that $r^{-2}$ belongs to $\mathcal{D}$ and fix $A \in\left(1, \frac{5}{4}\right)$, there is a zero Lebesgue measure subset $\mathcal{E}_{n, r, A} \subset(0,+\infty)$ such that the following holds for any $m \in(0,+\infty) \backslash \mathcal{E}_{n, r, A}$. For any large enough $s \gg 1$, for any couple of real-valued functions $\left(w_{0}, w_{1}\right) \in H^{s+2}\left(\mathbb{S}^{1} \times r \mathbb{S}^{1}\right) \times H^{s}\left(\mathbb{S}^{1} \times r \mathbb{S}^{1}\right)$ with $\left\|w_{0}\right\|_{H^{s+2}}+$ $\left\|w_{1}\right\|_{H^{s}}=1$, there are $C, K>0$ such that if $\varepsilon>0$ is small enough then the beam equation (1) admits a unique solution

$$
w \in \mathcal{C}^{0}\left(\left(-C \varepsilon^{-A n},+C \varepsilon^{-A n}\right), H^{s+2}\left(\mathbb{S}^{1} \times r \mathbb{S}^{1}\right)\right) \cap \mathcal{C}^{1}\left(\left(-C \varepsilon^{-A n},+C \varepsilon^{-A n}\right), H^{s}\left(\mathbb{S}^{1} \times r \mathbb{S}^{1}\right)\right),
$$

with initial data $(w(0), \dot{w}(0))=\left(\varepsilon w_{0}, \varepsilon w_{1}\right)$. Furthermore one has

$$
\forall t \in\left(-C \varepsilon^{-A n},+C \varepsilon^{-A n}\right) \quad\|w(t)\|_{H^{s+2}}+\|\dot{w}(t)\|_{H^{s}} \leq K \varepsilon .
$$

Remark 1.3. For instance, the previous result covers the case of the manifold $\mathbb{S}^{1} \times \sqrt[3]{2} \mathbb{S}^{1}$ thanks to the Roth theorem with with $r^{-2}=\frac{1}{\sqrt[3]{4}}$. It would be very interesting to understand if the analogue of Theorem 1.2 for the Klein-Gordon equation (2) is true or not. Although a modification of the condition $A \in\left(1, \frac{5}{4}\right)$ may be necessary, the proof would work for the semi-linear equation $\left(\partial_{t}^{2}+|\Delta|^{\alpha}+m^{2}\right) w=w^{n+1}$ where $|\Delta|^{\alpha}$ is a fractional power of the Laplace-Beltrami operator with $\alpha>1$. A similar phenomenon is studied in [5] by Bambusi-Sire for a Schrödinger equation. 
Although the strategy of the proof is quite similar of that of [26], we recall its main lines and explain several technical differences. The analysis of the equation begins by a reduction to the order one :

$$
\left(i \partial_{t}+\sqrt{\Delta^{2}+m^{2}}\right) u=w^{n+1}, \quad w:=\left(\Delta^{2}+m^{2}\right)^{-\frac{1}{2}} \operatorname{Re}(u),
$$

where we have introduced $u:=\left(-i \partial_{t}+\sqrt{\Delta^{2}+m^{2}}\right) w$ (remember that $w$ takes real values). The problem is equivalent to get a priori bounds on $\|u(t)\|_{H^{s}}$ once we assumed the initial condition $\|u(0)\|_{H^{s}}$ is of order $\varepsilon$. One easily proves the estimate $\frac{d}{d t}\|u(t)\|_{H^{s}}^{2}=\mathcal{O}\left(\left\|w^{n+1}\right\|_{H^{s}}\|u(t)\|_{H^{s}}\right)$ that leads to the a priori bound $\frac{d}{d t}\|u(t)\|_{H^{s}}^{2}=\mathcal{O}\left(\varepsilon^{2+n}\right)$. An integration around $t=0$ merely leads to the local existence time $\varepsilon^{-n}$. Let us roughly explain the strategy of the normal form to improve the local existence time (we also refer the reader to [20, pages 541-544]).

Using (4) and decomposing $w^{n+1}$ with the spectral projectors of $-\Delta$, we will construct four $(n+2)$ multilinear operator $\mathcal{M}_{\ell}, \widehat{\mathcal{M}}_{\ell}, \mathcal{R}, \widehat{\mathcal{R}}_{\ell}$ such that the derivative of the squared Sobolev norm $\|u(t)\|_{H^{s}}^{2}$ can be written as a sum

$$
\begin{aligned}
& \sum_{\ell=0}^{n} \operatorname{Re} i\left\langle\mathcal{M}_{\ell}(\underbrace{\bar{u}, \ldots, \bar{u}}_{\ell}, \underbrace{u, \ldots, u}_{n-\ell}) u, u\right\rangle+\sum_{\ell=0}^{n} \operatorname{Re} i\left\langle\mathcal{R}_{\ell}(\underbrace{\bar{u}, \ldots, \bar{u}}_{\ell}, \underbrace{u, \ldots, u}_{n-\ell}) u, u\right\rangle \\
& +\sum_{\ell=0}^{n} \operatorname{Re} i\left\langle\widehat{\mathcal{M}}_{\ell}(\underbrace{\bar{u}, \ldots, \bar{u}}_{\ell}, \underbrace{u, \ldots, u}_{n-\ell}) \bar{u}, u\right\rangle+\sum_{\ell=0}^{n} \operatorname{Re} i\left\langle\widehat{\mathcal{R}}_{\ell}(\underbrace{\bar{u}, \ldots, \bar{u}}_{\ell}, \underbrace{u, \ldots, u}_{n-\ell}) \bar{u}, u\right\rangle .
\end{aligned}
$$

A precise statement is given in Proposition 4.1. The idea is then to eliminate $\widehat{\mathcal{M}}_{\ell}, \mathcal{R}, \widehat{\mathcal{R}}_{\ell}$ by adding a higher order term $M(u(t))$ of $\|u(t)\|_{H^{s}}^{2}$. In other words, one has $M(u)=o\left(\varepsilon^{2}\right)$ and $\frac{d}{d t}\left[\|u(t)\|_{H^{s}}^{2}-M(u(t))\right]$ does not contain $\widehat{\mathcal{M}}_{\ell}, \mathcal{R}, \widehat{\mathcal{R}}_{\ell}$. A similar strategy is used to eliminate a part of the term $\mathcal{M}_{\ell}$ by a normal form procedure (the author has not succeeded to totally eliminate this term and this explains why the improved time is less than $\varepsilon^{-2 n}$, see 20, page 542]). Such a strategy leads to get an a priori estimate of the form $\frac{d}{d t}\left[\|u(t)\|_{H^{s}}^{2}-M(u(t))\right]=\mathcal{O}\left(\varepsilon^{2+A n}\right)$ with $A>1$. Note now that $\|u(t)\|_{H^{s}}^{2}$ and $\|u(t)\|_{H^{s}}^{2}-M(u(t))$ are both equivalent near 0 and are of order $\varepsilon^{2}$. An integration around $t=0$ leads to improve the local existence time to $\varepsilon^{-A n}$ (see the licit computations in Section 5).

Let us now explain several differences with [26.

- In the normal form procedure, one meets the following "small divisors issue" : one needs to estimate $\mathbb{Z}$-linear combinations of $n+2$ eigenvalues $\sqrt{\left(\lambda_{k}^{2}-1\right)^{2}+m^{2}}$ of $\sqrt{\Delta^{2}+m^{2}}$. Using the fact that $r^{-2}$ is Diophantine and the asymptotic $\lambda_{k} \simeq \sqrt{k}$, we get for every $m>0$ and $\aleph>1$

$$
\forall k_{1}>k_{2} \quad \sqrt{\left(\lambda_{k_{1}}^{2}-1\right)^{2}+m^{2}}-\sqrt{\left(\lambda_{k_{2}}^{2}-1\right)^{2}+m^{2}} \geq \frac{C(m, r, \aleph)}{\left(\lambda_{k_{1}}+\lambda_{k_{2}}\right)^{2 \aleph}} .
$$

Following a proof by Zhang [26, we will prove that for any positive number $\rho>0$, for almost all positive number $m>0$ (in the sense of Lebesgue), there is a real number $\nu_{0}=\nu_{0}(n, r, \rho, m)>0$ such that for any $\left(k_{0}, \ldots, k_{n+1}\right) \in(\mathbb{N} \backslash\{0\})^{n+2}$ the following bound from below holds true :

$$
\left|\sum_{\ell=0}^{n+1} \pm \sqrt{\left(\lambda_{k_{\ell}}^{2}-1\right)^{2}+m^{2}}\right| \geq \frac{C(m, n, r, \rho)}{\left(\lambda_{k_{0}}+\lambda_{k_{n+1}}\right)^{4+\rho} \max \left(\lambda_{k_{1}}, \ldots, \lambda_{k_{n}}\right)^{\nu_{0}}} .
$$

except of course if the left-hand side of (6) vanishes. Such an inequality is useful to eliminate a part of the nonlinearity $w^{n+1}$ in the beam equation (1). In the papers [26, 11], one has the inclusion

$$
\left\{\lambda_{k}, k \geq 1\right\} \subset\{\sqrt{k}, k \geq 1\},
$$

which implies that $\lambda_{k+1}^{2}-\lambda_{k}^{2}$ is bounded from below. In our work, we merely has the weaker estimate (see Lemma 2.1)

$$
\forall \aleph>1 \quad \forall k \geq 1 \quad \lambda_{k+1}^{2}-\lambda_{k}^{2} \geq C(\aleph) k^{-\aleph}
$$

and the small divisors estimates $(6)$ is the best the author succeeded to get. This is crucial to compute the supremum of numbers $A>1$ such that the conclusion of Theorem 1.2 holds true. We have to keep 
in mind the following rule : the smaller the exponent of $\left(\lambda_{k_{0}}+\lambda_{k_{n+1}}\right)$ is, the better the improvement of the local existence time can be.

- The second point we want to stress is the reason why we are not able to deal with the Klein-Gordon equation on an irrational torus $\mathbb{S}^{1} \times r \mathbb{S}^{1}$. To understand the interaction of the nonlinearity $w^{n+1}$ with the linear part, the normal form procedure needs to prove that some multilinear operators, used to eliminate $\mathcal{M}_{\ell}, \widehat{\mathcal{M}}_{\ell}, \mathcal{R}, \widehat{\mathcal{R}}_{\ell}\left(\right.$ see $(5)$ ), are bounded on the Sobolev space $H^{s}\left(\mathbb{S}^{1} \times r \mathbb{S}^{1}\right)$. In [26, line (2.1.9)], the following estimates, proven thanks to (7), are used to get the boundedness on the Sobolev spaces :

$$
\forall \omega>2 \quad \forall a \geq 1 \quad \forall \ell \geq 1 \quad \sum_{k \geq 1} \frac{1}{\left(\left|\lambda_{k}-\lambda_{\ell}\right|+a\right)^{\omega}} \leq \frac{C \lambda_{\ell}}{a^{\omega-2}} .
$$

To our knowledge, it is not clear whether a sequence $\left(\lambda_{k}\right)_{k \geq 1}$ which is badly separated in the sense of (8) can satisfy (9). In our work, Lemma 3.4 will only give the weaker version

$$
\forall \omega>2 \quad \forall a \geq 1 \quad \forall \ell \geq 1 \quad \sum_{k \geq 1} \frac{1}{\left(\left|\lambda_{k}-\lambda_{\ell}\right|+a\right)^{\omega}} \leq \frac{C \lambda_{\ell}^{2}}{a^{\omega-2}}
$$

This simple fact unfortunately forbids in our approach to consider the Klein-Gordon equation. However, the beam equation admits a regularizing effect that allows us to deal with this issue. Let us recall that in the framework of the Klein-Gordon equation $\left(\partial_{t}^{2}-\Delta+m^{2}\right) w=w^{n+1}$ with $u$ replaced by $\left(-i \partial_{t}+\sqrt{-\Delta+m^{2}}\right) w$, the nonlinearity becomes $\left[\left(-\Delta+m^{2}\right)^{-\frac{1}{2}} \operatorname{Re}(u)\right]^{n+1}$. For the beam equation, the fact that the operator $\left(\Delta^{2}+m^{2}\right)^{-\frac{1}{2}}$ is more regularizing than $\left(-\Delta+m^{2}\right)^{-\frac{1}{2}}$ gives a little gain of derivatives which counterbalances the multiplicative lost $\lambda_{\ell}^{2}$ in $(10)$. Consequently we will be able to eliminate, totally or partially, the operators $\mathcal{M}_{\ell}, \widehat{\mathcal{M}}_{\ell}, \mathcal{R}, \widehat{\mathcal{R}}_{\ell}$. We finally conclude by obtaining better a priori estimates.

In Section 2, we sum up the spectral analysis we need for our purpose (the asymptotic behavior of the eigenvalues, the multilinear estimates of the spectral projectors and the small divisors estimates proved in Section 6). Sections 3 and 4 are devoted to the analysis of the nonlinearity with the aid of specific multilinear operators (we follow the same scheme of proof than that of [26] and thus we skip several similarities). Theorem 1.2 is proven in Section 5.

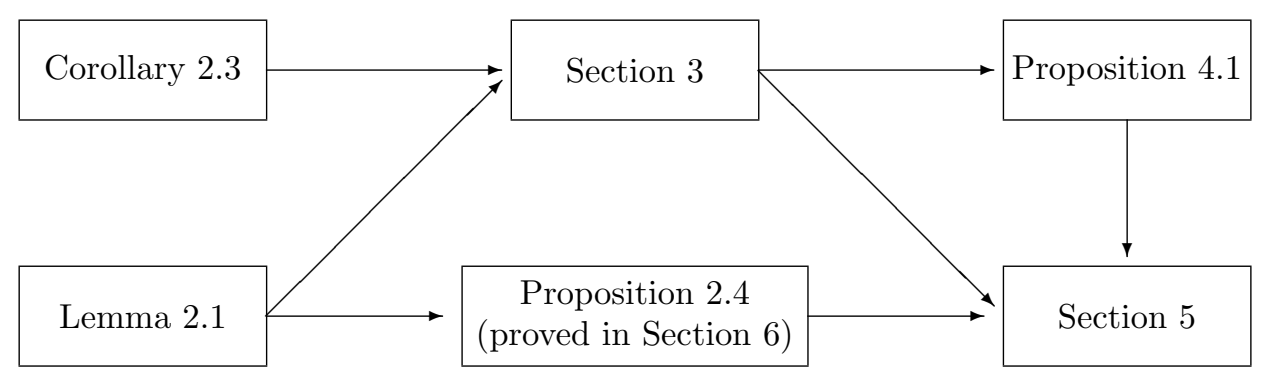

To make shorter several formulas, it will be convenient to write $H^{s}$ instead of $H^{s}\left(\mathbb{S}^{1} \times r \mathbb{S}^{1}\right)$.

\section{Spectral analysis}

\subsection{Spectrum and universal multilinear estimates}

In all the paper, we assume that $r^{-2}$ belongs to the set $\mathcal{D}$ (see Definition 1.1). The spectrum of the operator $-\Delta$ on $L^{2}\left(\mathbb{S}^{1} \times r \mathbb{S}^{1}\right)$ is pure point and is given by

$$
\operatorname{Sp}(-\Delta)=\left\{p^{2}+r^{-2} q^{2},(p, q) \in \mathbb{Z}^{2}\right\}=\left\{p^{2}+r^{-2} q^{2},(p, q) \in \mathbb{N}^{2}\right\} .
$$


We sort without counting multiplicities $\operatorname{Sp}(-\Delta+1)$ as an increasing sequence $\left(\lambda_{k}^{2}\right)_{k \geq 1}$ with $\lambda_{k} \geq 0$. For instance, one has $\lambda_{0}=1$.

Lemma 2.1. Fix any $\sigma>\frac{3}{2}$ and $\aleph>1$, the following asymptotics hold as $k$ tends to infinity :

$$
\begin{aligned}
\lambda_{k} & \simeq \sqrt{k}, \\
\lambda_{k+1}-\lambda_{k} & \gtrsim k^{-\sigma}, \\
\lambda_{k+1}^{2}-\lambda_{k}^{2} & \gtrsim k^{-\aleph},
\end{aligned}
$$

where the symbols $\gtrsim$ and $\simeq$ involve constants which may depend on $(\sigma, \aleph, r)$.

Proof. The proof of (11) uses a basic Weyl law argument. We begin by writing for any positive and real number $N$ :

$$
\begin{gathered}
\#\left\{(p, q) \in \mathbb{N}^{2}, p^{2}+r^{-2} q^{2} \leq N\right\}-\sum_{q=0}^{\lfloor r \sqrt{N}\rfloor} 1+\left\lfloor\sqrt{N-r^{-2} q^{2}}\right\rfloor=0 \\
\left|\#\left\{(p, q) \in \mathbb{N}^{2}, p^{2}+r^{-2} q^{2} \leq N\right\}-N r \times \frac{1}{r \sqrt{N}} \sum_{q=0}^{\lfloor r \sqrt{N}\rfloor} \sqrt{1-\left(\frac{q}{r \sqrt{N}}\right)^{2}}\right| \leq C(r) \sqrt{N} .
\end{gathered}
$$

Recognizing a modified Riemann sum and using the irrationality of $r^{-2}$, we get the following asymptotics as $N$ tends to infinity :

$$
\begin{aligned}
\#\left\{(p, q) \in \mathbb{N}^{2}, p^{2}+r^{-2} q^{2} \leq N\right\} & \sim N r \int_{0}^{1} \sqrt{1-\gamma^{2}} d \gamma \\
& \sim \frac{r \pi}{4} N \\
\#\left\{k \in \mathbb{N}^{\star}, \lambda_{k}^{2}-1 \leq N\right\} & \sim \frac{r \pi}{4} N \\
k & \sim \frac{r \pi}{4} \lambda_{k}^{2} .
\end{aligned}
$$

To see 12 and 13 , we write

$$
\lambda_{k+1}^{2}=1+P^{2}+r^{-2} Q^{2}>\lambda_{k}^{2}=1+p^{2}+r^{-2} q^{2}
$$

where $P, Q, p$ and $q$ are integers. If $q=Q$ holds, then $\lambda_{k+1}^{2}-\lambda_{k}^{2} \geq 1$ obviously holds and we get $\lambda_{k+1}-\lambda_{k} \gtrsim \frac{1}{\sqrt{k}}$ thanks to [11. If $q \neq Q$ holds, we remember Definition 1.1 and we get for any $\mu>2$

$$
\begin{aligned}
\lambda_{k+1}^{2}-\lambda_{k}^{2} & =\left(P^{2}-p^{2}\right)+r^{-2}\left(Q^{2}-q^{2}\right) \\
& \gtrsim \frac{1}{\left|Q^{2}-q^{2}\right|^{\mu-1}} \geq \frac{1}{\left|Q^{2}+q^{2}\right|^{\mu-1}} \gtrsim \frac{1}{\lambda_{k+1}^{2 \mu-2}}, \\
\lambda_{k+1}-\lambda_{k} & \gtrsim \frac{1}{\lambda_{k}^{2 \mu-1}} \gtrsim \frac{1}{k^{\mu-\frac{1}{2}}} .
\end{aligned}
$$

\subsection{Multilinear estimates}

For any integer $k \geq 1$, let us denote by $\Pi_{k}$ the spectral projector of $L^{2}\left(\mathbb{S}^{1} \times r \mathbb{S}^{1}\right)$ on $\operatorname{ker}\left(1-\Delta-\lambda_{k}^{2}\right)$. We also denote by $\mathcal{D}\left(\mathbb{S}^{1} \times r \mathbb{S}^{1}\right)$ the vector space of smooth functions on $\mathbb{S}^{1} \times r \mathbb{S}^{1}$. We will make use of universal multilinear estimates proven by Delort and Szeftel (10, Proposition 1.2.1 and Proposition 1.2.2]). It turns out that the following result is true on any compact manifold without boundary but the proof is elementary if one knows the eigenfunctions as it is the case for tori. 
Proposition 2.2. Consider two integers $n \geq 2$ and $N \geq 1$. For any real number $\nu>n$, there is a positive constant $C(N, \nu, n, r)$ such that for any $u_{0}, \ldots, u_{n+1} \in \mathcal{D}\left(\mathbb{S}^{1} \times r \mathbb{S}^{1}\right)$, any nonnegative integers $k_{1} \leq \cdots \leq k_{n+1} \leq k_{0}$ and $N \in \mathbb{N}^{\star}$ one has

$$
\left|\int_{\mathbb{S}^{1} \times r \mathbb{S}^{1}} \Pi_{k_{0}}\left(u_{0}\right) \ldots \Pi_{k_{n+1}}\left(u_{n+1}\right) d x d y\right| \leq C(N, \nu, n, r) \lambda_{k_{n}}^{\nu}\left(1+\frac{\lambda_{k_{0}}-\lambda_{k_{n+1}}}{\lambda_{k_{n}}}\right)^{-N} \prod_{j=0}^{n+1}\left\|u_{j}\right\|_{L^{2}\left(\mathbb{S}^{1} \times r \mathbb{S}^{1}\right)},
$$

where $d x d y$ is the Riemannian measure on $\mathbb{S}^{1} \times r \mathbb{S}^{1}$.

Proof. It is clear that it suffices to assume that each $u_{j}$ belongs to the range of $\Pi_{k_{j}}$ for any integer $j \in[0, n+1]$. By writing $\lambda_{k_{j}}^{2}-1=p_{j}^{2}+r^{-2} q_{j}^{2}$ with $\left(p_{j}, q_{j}\right) \in \mathbb{N}^{2}$ and remembering that $r^{-2}$ is irrational, we can write $u_{j}(x, y)$, with $(x, y) \in \mathbb{R}^{2} /(2 \pi \mathbb{Z} \times 2 \pi r \mathbb{Z})$, as a sum of at most four simple functions of the form $\exp \left( \pm i p_{j} x \pm i q_{j} \frac{y}{r}\right)$ and the left-hand side of $(15)$ is a sum of at most $4^{n+2}$ integrals of products of the above trigonometric functions. Thus, if the left-hand side of 15 is not zero then there are numbers $\tau_{0}, \tau_{0}^{\prime}, \ldots, \tau_{n+1}, \tau_{n+1}^{\prime} \in\{ \pm 1\}$ such that

$$
\int_{\mathbb{S}^{1} \times r \mathbb{S}^{1}} \exp \left(i\left(\tau_{0} p_{0}+\cdots+\tau_{n+1} p_{n+1}\right) x+i\left(\tau_{0}^{\prime} q_{0}+\cdots+\tau_{n+1}^{\prime} q_{n+1}\right) \frac{y}{r}\right) d x d y \neq 0
$$

In other words, we have

$$
\begin{aligned}
& \tau_{0} p_{0}+\cdots+\tau_{n+1} p_{n+1}=0 \\
& \tau_{0}^{\prime} q_{0}+\cdots+\tau_{n+1}^{\prime} q_{n+1}=0 .
\end{aligned}
$$

In particular, we deduce

$$
\left|p_{0}-p_{n+1}\right|+\left|q_{0}-q_{n+1}\right| \leq \sum_{j=1}^{n} p_{j}+q_{j} \leq C(r) n \lambda_{k_{n}} .
$$

Hence, using the property that $(x, y) \mapsto \sqrt{1+x^{2}+r^{-2} y^{2}}$ is a Lipschitz function on $[0,+\infty)^{2}$, we get

$$
\lambda_{k_{0}}-\lambda_{k_{n+1}} \leq C(r) n \lambda_{k_{n}} .
$$

It is now easy to prove $(15)$ by using a Hölder inequality and the Sobolev embedding $H^{\frac{\nu}{n}}\left(\mathbb{S}^{1} \times r \mathbb{S}^{1}\right) \subset$ $L^{\infty}\left(\mathbb{S}^{1} \times r \mathbb{S}^{1}\right)$. Indeed, the left-hand side of $(15)$ is less than or equal to

$$
\left\|u_{0}\right\|_{L^{2}\left(\mathbb{S}^{1} \times r \mathbb{S}^{1}\right)}\left\|u_{n+1}\right\|_{L^{2}\left(\mathbb{S}^{1} \times r \mathbb{S}^{1}\right)} \prod_{j=1}^{n}\left\|u_{j}\right\|_{L^{\infty}\left(\mathbb{S}^{1} \times r \mathbb{S}^{1}\right)} \leq C\left(\frac{\nu}{n}, r\right)^{n} \lambda_{k_{n}}^{\nu} \prod_{j=0}^{n+1}\left\|u_{j}\right\|_{L^{2}\left(\mathbb{S}^{1} \times r \mathbb{S}^{1}\right)} .
$$

The conclusion comes from 16 .

In the same spirit that in the paper [26], we will indeed use the following corollary.

Corollary 2.3. Let us consider $\delta \in(0,1)$ such that $\frac{1}{\delta}-\delta^{2}<1$ and $\frac{1-\delta^{2}}{\delta}<1$ hold 1 . Consider moreover two integers $n \geq 1$ and $N \geq 1$. For any real number $\nu>n$, there is a positive number $C=C(N, \nu, n, r, \delta)$ such that for any $\left(k_{0}, \ldots, k_{n+1}\right) \in(\mathbb{N} \backslash\{0\})^{n+2}$ with $\max \left(k_{1}, \ldots, k_{n}\right) \leq k_{n+1}$ and for any $\left(u_{1}, \ldots, u_{n+1}\right) \in$ $\mathcal{D}\left(\mathbb{S}^{1} \times r \mathbb{S}^{1}\right)^{n+1}$ the following two assertions hold

i) if $\frac{\lambda_{k_{0}}}{\lambda_{k_{n+1}}} \in\left[\delta, \frac{1}{\delta}\right]$ holds then one has

$$
\begin{aligned}
& \left\|\Pi_{k_{0}}\left(\Pi_{k_{1}}\left(u_{1}\right) \ldots \Pi_{k_{n+1}}\left(u_{n+1}\right)\right)\right\|_{L^{2}\left(\mathbb{S}^{1} \times r \mathbb{S}^{1}\right)} \\
& \quad \leq C \max \left(\lambda_{k_{1}}, \ldots, \lambda_{k_{n}}\right)^{\nu}\left(1+\frac{\left|\lambda_{k_{0}}-\lambda_{k_{n+1}}\right|}{\max \left(\lambda_{k_{1}}, \ldots, \lambda_{k_{n}}\right)}\right)^{-N} \prod_{j=1}^{n+1}\left\|u_{j}\right\|_{L^{2}\left(\mathbb{S}^{1} \times r \mathbb{S}^{1}\right)} .
\end{aligned}
$$

\footnotetext{
${ }^{1}$ any number $\delta$ close enough to 1 is convenient.
} 
ii) if $\frac{\lambda_{k_{0}}}{\lambda_{k_{n+1}}} \notin\left[\delta, \frac{1}{\delta}\right]$ holds or if $\max \left(\lambda_{k_{1}}, \ldots, \lambda_{k_{n}}\right)>\delta^{2} \lambda_{k_{n+1}}$ holds, then one has

$$
\left\|\Pi_{k_{0}}\left(\Pi_{k_{1}}\left(u_{1}\right) \ldots \Pi_{k_{n+1}}\left(u_{n+1}\right)\right)\right\|_{L^{2}\left(\mathbb{S}^{1} \times r \mathbb{S}^{1}\right)} \leq C \frac{\max _{2}\left(\lambda_{k_{1}}, \ldots, \lambda_{k_{n+1}}\right)^{\nu+N}}{\left(\lambda_{k_{0}}+\cdots+\lambda_{k_{n+1}}\right)^{N}} \prod_{j=1}^{n+1}\left\|u_{j}\right\|_{L^{2}\left(\mathbb{S}^{1} \times r \mathbb{S}^{1}\right)},
$$

where $\max _{2}\left(\lambda_{k_{1}}, \ldots, \lambda_{k_{n+1}}\right)$ is the second largest number among $\lambda_{k_{1}}, \ldots, \lambda_{k_{n+1}}$.

Proof. i) We begin by assuming $k_{0} \leq \max \left(k_{1}, \ldots, k_{n}\right) \leq k_{n+1}$. The third largest number among $\lambda_{k_{0}}, \ldots, \lambda_{k_{n+1}}$ is of the same order than $\lambda_{k_{n+1}}, \lambda_{k_{0}}$ and $\lambda_{\max \left(k_{1}, \ldots, k_{n}\right)}$. Thus, we can write

$$
\left|\lambda_{k_{0}}-\lambda_{k_{n+1}}\right| \leq \lambda_{k_{0}}+\lambda_{k_{n+1}} \leq\left(1+\frac{1}{\delta}\right) \lambda_{k_{0}} \leq\left(1+\frac{1}{\delta}\right) \max \left(\lambda_{k_{1}}, \ldots, \lambda_{k_{n}}\right) .
$$

We can bound $\left(1+\frac{\lambda_{k_{0}}-\lambda_{k_{n+1}}}{\lambda_{k_{n}}}\right)^{-N}$ by 1 in 15 and we get

$$
\begin{aligned}
\left\|\Pi_{k_{0}}\left(\Pi_{k_{1}}\left(u_{1}\right) \ldots \Pi_{k_{n+1}}\left(u_{n+1}\right)\right)\right\|_{L^{2}\left(\mathbb{S}^{1} \times r \mathbb{S}^{1}\right)} & =\sup _{u_{0} \neq 0} \frac{1}{\left\|u_{0}\right\|_{L^{2}\left(\mathbb{S}^{1} \times r \mathbb{S}^{1}\right)}}\left|\int_{X} u_{0} \Pi_{k_{0}}\left(\Pi_{k_{1}}\left(u_{1}\right) \ldots \Pi_{k_{n+1}}\left(u_{n+1}\right)\right) d x\right| \\
& =\sup _{u_{0} \neq 0} \frac{1}{\left\|u_{0}\right\|_{L^{2}\left(\mathbb{S}^{1} \times r \mathbb{S}^{1}\right)}}\left|\int_{X} \Pi_{k_{0}}\left(u_{0}\right) \ldots \Pi_{k_{n+1}}\left(u_{n+1}\right) d x\right| \\
& \lesssim \max \left(\lambda_{k_{1}}, \ldots, \lambda_{k_{n}}\right)^{\nu} \prod_{j=1}^{n+1}\left\|u_{j}\right\|_{L^{2}\left(\mathbb{S}^{1} \times r \mathbb{S}^{1}\right)} .
\end{aligned}
$$

Using (19), we obtain (17). If $k_{0}>\max \left(k_{1}, \ldots, k_{n}\right)$ holds, then (17) is nothing else than (15).

ii) Let us explain why there exists $\varrho \in(0,1)$ (independent of $k_{0}, \ldots, k_{n+1}$ ) such that the following inequality is true :

$$
\max _{2}\left(\lambda_{k_{0}}, \ldots, \lambda_{k_{n+1}}\right)-\max \left(\lambda_{k_{1}}, \ldots, \lambda_{k_{n}}\right) \leq \varrho \max \left(\lambda_{k_{0}}, \ldots, \lambda_{k_{n}}\right) .
$$

Note that the inequality $0 \leq \max _{2}\left(\lambda_{k_{0}}, \ldots, \lambda_{k_{n+1}}\right)-\max \left(\lambda_{k_{1}}, \ldots, \lambda_{k_{n}}\right)$ obviously holds. We now consider several subcases :

- if $\frac{\lambda_{k_{0}}}{\lambda_{k_{n+1}}}>\frac{1}{\delta}$ holds then we have $\max _{2}\left(\lambda_{k_{0}}, \ldots, \lambda_{k_{n+1}}\right)=\lambda_{k_{n+1}}$ and $\max \left(\lambda_{k_{0}}, \ldots, \lambda_{k_{n+1}}\right)=\lambda_{k_{0}}$. The number $\varrho=\delta$ is convenient.

- if $\frac{\lambda_{k_{n+1}}}{\lambda_{k_{0}}}>\frac{1}{\delta}$ holds then we have $\max \left(\lambda_{k_{0}}, \ldots, \lambda_{k_{n+1}}\right)=\lambda_{k_{n+1}}$. We should make a discussion about the position of $\lambda_{k_{0}}$. If $\lambda_{k_{0}} \leq \max \left(\lambda_{k_{1}}, \ldots, \lambda_{k_{n}}\right) \leq \lambda_{k_{n+1}}$ holds then the left-hand side of (21) vanishes. If $\max \left(\lambda_{k_{1}}, \ldots, \lambda_{k_{n}}\right) \leq \lambda_{k_{0}} \leq \lambda_{k_{n+1}}$ then one just have to write

$$
\max _{2}\left(\lambda_{k_{0}}, \ldots, \lambda_{k_{n+1}}\right)-\max \left(\lambda_{k_{1}}, \ldots, \lambda_{k_{n}}\right)=\lambda_{k_{0}}-\max \left(\lambda_{k_{1}}, \ldots, \lambda_{k_{n}}\right) \leq \lambda_{k_{0}} \leq \delta \lambda_{k_{n+1}} .
$$

- let us assume that $\frac{\lambda_{k_{0}}}{\lambda_{k_{n+1}}}$ belongs to $\left[\delta, \frac{1}{\delta}\right], \max \left(\lambda_{k_{1}}, \ldots, \lambda_{k_{n}}\right)>\delta^{2} \lambda_{k_{n+1}}$ and $\max \left(\lambda_{k_{1}}, \ldots, \lambda_{k_{n}}\right) \leq$ $\lambda_{k_{n+1}} \leq \lambda_{k_{0}}$ hold. We then have

$$
\max _{2}\left(\lambda_{k_{0}}, \ldots, \lambda_{k_{n+1}}\right)-\max \left(\lambda_{k_{1}}, \ldots, \lambda_{k_{n}}\right) \leq\left(1-\delta^{2}\right) \lambda_{k_{n+1}} \leq \frac{1}{\delta}\left(1-\delta^{2}\right) \lambda_{k_{0}} .
$$

The previous gives 21] because we have assumed that $\frac{1-\delta^{2}}{\delta}$ is less than 1 .

- let us assume that $\frac{\lambda_{k_{0}}}{\lambda_{k_{n+1}}}$ belongs to $\left[\delta, \frac{1}{\delta}\right], \max \left(\lambda_{k_{1}}, \ldots, \lambda_{k_{n}}\right)>\delta^{2} \lambda_{k_{n+1}}$ and $\max \left(\lambda_{k_{1}}, \ldots, \lambda_{k_{n}}\right) \leq \lambda_{k_{0}} \leq$ $\lambda_{k_{n+1}}$ hold. Thus, we get

$$
\max _{2}\left(\lambda_{k_{0}}, \ldots, \lambda_{k_{n+1}}\right)-\max \left(\lambda_{k_{1}}, \ldots, \lambda_{k_{n}}\right) \leq \lambda_{k_{0}}-\delta^{2} \lambda_{k_{n+1}} \leq\left(\frac{1}{\delta}-\delta^{2}\right) \lambda_{k_{n+1}} .
$$


- we finally assume that $\frac{\lambda_{k_{0}}}{\lambda_{k_{n+1}}}$ belongs to $\left[\delta, \frac{1}{\delta}\right], \max \left(\lambda_{k_{1}}, \ldots, \lambda_{k_{n}}\right)>\delta^{2} \lambda_{k_{n+1}}$ and $\lambda_{k_{0}} \leq \max \left(\lambda_{k_{1}}, \ldots, \lambda_{k_{n}}\right) \leq$ $\lambda_{k_{n+1}}$ hold. Such a case is obvious because the left-hand side of (21) vanishes.

The inequality (21) is proven. Let us prove (18). We note now that we have $\max _{3}\left(\lambda_{k_{0}}, \ldots, \lambda_{k_{n+1}}\right) \leq$ $\max _{2}\left(\lambda_{k_{1}}, \ldots, \lambda_{k_{n+1}}\right)=\max \left(\lambda_{k_{1}}, \ldots, \lambda_{k_{n}}\right)$ where $\max _{3}\left(\lambda_{k_{0}}, \ldots, \lambda_{k_{n+1}}\right)$ is the third largest number among $\lambda_{k_{0}}, \ldots, \lambda_{k_{n+1}}$. By combining with 21), that gives us

$$
\begin{aligned}
\frac{\lambda_{k_{0}}+\cdots+\lambda_{k_{n+1}}}{\max _{2}\left(\lambda_{k_{1}}, \ldots, \lambda_{k_{n+1}}\right)} & \lesssim \frac{\max \left(\lambda_{k_{0}}, \ldots, \lambda_{k_{n+1}}\right)}{\max _{2}\left(\lambda_{k_{1}}, \ldots, \lambda_{k_{n+1}}\right)} \\
& \lesssim \frac{\max \left(\lambda_{k_{0}}, \ldots, \lambda_{k_{n+1}}\right)-\max _{2}\left(\lambda_{k_{0}}, \ldots, \lambda_{k_{n+1}}\right)+\max \left(\lambda_{k_{1}}, \ldots, \lambda_{k_{n}}\right)}{\max _{2}\left(\lambda_{k_{1}}, \ldots, \lambda_{k_{n+1}}\right)} \\
& \lesssim 1+\frac{\max \left(\lambda_{k_{0}}, \ldots, \lambda_{k_{n+1}}\right)-\max _{2}\left(\lambda_{k_{0}}, \ldots, \lambda_{k_{n+1}}\right)}{\max _{3}\left(\lambda_{k_{0}}, \ldots, \lambda_{k_{n+1}}\right)}
\end{aligned}
$$

From 15 and 200 , we get the conclusion.

\subsection{Small divisors}

For any $m>0$ and $\ell \in[0, n] \cap \mathbb{N}$, let us define the following two maps $F_{m}^{\ell}$ and $\widehat{F}_{m}^{\ell}$ on $[1,+\infty)^{2 n+2}$ :

$$
\begin{gathered}
F_{m}^{\ell}\left(\xi_{0}, \ldots, \xi_{n+1}\right)=\sum_{j=0}^{\ell} \sqrt{\left(\xi_{j}^{2}-1\right)^{2}+m^{2}}-\sum_{j=\ell+1}^{n+1} \sqrt{\left(\xi_{j}^{2}-1\right)^{2}+m^{2}}, \\
\widehat{F}_{m}^{\ell}\left(\xi_{0}, \ldots, \xi_{n+1}\right)=\sum_{j=0}^{\ell} \sqrt{\left(\xi_{j}^{2}-1\right)^{2}+m^{2}}-\sum_{j=\ell+1}^{n} \sqrt{\left(\xi_{j}^{2}-1\right)^{2}+m^{2}}+\sqrt{\left(\xi_{n+1}^{2}-1\right)^{2}+m^{2}} .
\end{gathered}
$$

We also define some specific subsets of $(\mathbb{N} \backslash\{0\})^{n+2}$ :

$$
\begin{aligned}
& \Omega_{n+2}(\ell):=\left\{\left(k_{0}, \ldots, k_{n+1}\right), \quad\left\{k_{0}, \ldots, k_{\ell}\right\}=\left\{k_{\ell+1}, \ldots, k_{n+1}\right\}\right\} \\
& \Omega_{n+2}(\widehat{\ell}):=\left\{\left(k_{0}, \ldots, k_{n+1}\right), \quad\left\{k_{n+1}, k_{0}, \ldots, k_{\ell}\right\}=\left\{k_{\ell+1}, \ldots, k_{n}\right\}\right\} .
\end{aligned}
$$

Note that the previous definitions are relevant only if $n$ is even. Note also that for any $k \in \Omega_{n+2}(\ell)$ one has $F_{m}^{\ell}\left(\lambda_{k_{0}}, \ldots, \lambda_{k_{n+1}}\right)=0$. The purpose of the next result is to explain that, for a generic $m>0$ and for any $k \notin \Omega_{n+2}(\ell)$, the number $\left|F_{m}^{\ell}\left(\lambda_{k_{0}}, \ldots, \lambda_{k_{n+1}}\right)\right|$ is bounded from below.

Proposition 2.4. For any positive number $\rho>0$, for almost every $m>0$ (in the sense of Lebesgue), any integer $\ell \in[0, n]$, there are $C>0$ and $\nu_{0}>0$ such that for all $\left(k_{0}, \ldots, k_{n+1}\right) \in(\mathbb{N} \backslash\{0\})^{n+2} \backslash \Omega_{n+2}(\ell)$ we have the following estimates

$$
\begin{aligned}
& \frac{1}{\left|F_{m}^{\ell}\left(\lambda_{k_{0}}, \ldots, \lambda_{k_{n+1}}\right)\right|} \leq C\left(\lambda_{k_{0}}+\lambda_{k_{n+1}}\right)^{4+\rho} \max \left(\lambda_{k_{1}}, \ldots, \lambda_{k_{n}}\right)^{\nu_{0}}, \\
& \frac{1}{\left|F_{m}^{\ell}\left(\lambda_{k_{0}}, \ldots, \lambda_{k_{n+1}}\right)\right|} \leq C\left(\lambda_{k_{0}}+\cdots+\lambda_{k_{n+1}}\right)^{\nu_{0}} .
\end{aligned}
$$

In the same spirit, if $\left(k_{0}, \ldots, k_{n+1}\right) \in(\mathbb{N} \backslash\{0\})^{n+2} \backslash \Omega_{n+2}(\widehat{\ell})$ then we have

$$
\begin{gathered}
\frac{1}{\left|\widehat{F}_{m}^{\ell}\left(\lambda_{k_{0}}, \ldots, \lambda_{k_{n+1}}\right)\right|} \leq C \frac{\left(\lambda_{k_{1}}+\cdots+\lambda_{k_{n}}\right)^{\nu_{0}}}{\lambda_{k_{0}}+\lambda_{k_{n+1}}}, \\
\frac{1}{\left|\widehat{F}_{m}^{\ell}\left(\lambda_{k_{0}}, \ldots, \lambda_{k_{n+1}}\right)\right|} \leq C\left(\lambda_{k_{0}}+\cdots+\lambda_{k_{n+1}}\right)^{\nu_{0}} .
\end{gathered}
$$


Remark 2.5. It is obvious that (26) is stronger than (27). However, the proof of (26) will be a consequence of (27).

Remark 2.6. We will see at the end of the proof that the number $\rho>0$ in Proposition 2.4 is linked to the number $A \in\left(1, \frac{5}{4}\right)$ of Theorem 1.2 by the formula $A=1+\frac{1}{4+\rho}$.

The proof of Proposition 2.4 uses in an essential way Lemma 2.1 and is adapted from 26, Theorem 2.3.1 and Proposition 2.3.6] and [7, Part 2.1]. We postpone the proof in Section [6 because it is quite technical.

\section{Multilinear operators}

In this part, we merely use the asymptotic $\lambda_{k} \simeq \sqrt{k}$. As usual, we respectively denote by $\mathcal{D}^{\prime}\left(\mathbb{S}^{1} \times r \mathbb{S}^{1}\right)$ and $\mathcal{L}\left(\mathcal{D}\left(\mathbb{S}^{1} \times r \mathbb{S}^{1}\right), \mathcal{D}^{\prime}\left(\mathbb{S}^{1} \times r \mathbb{S}^{1}\right)\right)$ the vector spaces of the distributions on $\mathbb{S}^{1} \times r \mathbb{S}^{1}$ and of the linear operators from $\mathcal{D}\left(\mathbb{S}^{1} \times r \mathbb{S}^{1}\right)$ to $\mathcal{D}^{\prime}\left(\mathbb{S}^{1} \times r \mathbb{S}^{1}\right)$. In the sequel we give sufficient conditions on $n$-multilinear operators $\mathcal{M}: \mathcal{D}\left(\mathbb{S}^{1} \times r \mathbb{S}^{1}\right)^{n} \rightarrow \mathcal{L}\left(\mathcal{D}\left(\mathbb{S}^{1} \times r \mathbb{S}^{1}\right), \mathcal{D}^{\prime}\left(\mathbb{S}^{1} \times r \mathbb{S}^{1}\right)\right)$ so that they admit a bounded extension from $\left(H^{s}\left(\mathbb{S}^{1} \times r \mathbb{S}^{1}\right)\right)^{n}$ to $\mathcal{L}\left(H^{s}\left(\mathbb{S}^{1} \times r \mathbb{S}^{1}\right), H^{-s^{\prime}}\left(\mathbb{S}^{1} \times r \mathbb{S}^{1}\right)\right)$ for some real number $s^{\prime}$.

Definition 3.1. Let us consider $\tau>0, \nu>0, \delta \in(0,1)$ and $n \in \mathbb{N}^{\star}$. We say that a multilinear operator $\mathcal{M}: \mathcal{D}\left(\mathbb{S}^{1} \times r \mathbb{S}^{1}\right)^{n} \rightarrow \mathcal{L}\left(\mathcal{D}\left(\mathbb{S}^{1} \times r \mathbb{S}^{1}\right), \mathcal{D}^{\prime}\left(\mathbb{S}^{1} \times r \mathbb{S}^{1}\right)\right)$ belongs to $\mathcal{M}_{n, \delta}^{\tau, \nu}$ if for every $N>1$ one can find a constant $C>0$ such that for any $\left(u_{1}, \ldots, u_{n+1}\right) \in \mathcal{D}\left(\mathbb{S}^{1} \times r \mathbb{S}^{1}\right)^{n+1}$ one has

i) if $\delta \leq \frac{\lambda_{k_{0}}}{\lambda_{k_{n+1}}} \leq \frac{1}{\delta}$ and $\max \left(k_{1}, \ldots, k_{n}\right) \leq k_{n+1}$ hold then

$$
\begin{aligned}
& \left\|\Pi_{k_{0}}\left(\mathcal{M}\left(\Pi_{k_{1}} u_{1}, \ldots, \Pi_{k_{n}} u_{n}\right) \Pi_{k_{n+1}} u_{n+1}\right)\right\|_{L^{2}\left(\mathbb{S}^{1} \times r \mathbb{S}^{1}\right)} \\
& \quad \leq C \lambda_{k_{0}}^{\tau} \max \left(\lambda_{k_{1}}, \ldots, \lambda_{k_{n}}\right)^{\nu}\left(1+\frac{\left|\lambda_{k_{0}}-\lambda_{k_{n+1}}\right|}{\max \left(\lambda_{k_{1}}, \ldots, \lambda_{k_{n}}\right)}\right)^{-N} \prod_{j=1}^{n+1}\left\|u_{j}\right\|_{L^{2}\left(\mathbb{S}^{1} \times r \mathbb{S}^{1}\right)} .
\end{aligned}
$$

ii) for all other frequencies, one has $\Pi_{k_{0}}\left(\mathcal{M}\left(\Pi_{k_{1}} u_{1}, \ldots, \Pi_{k_{n}} u_{n}\right) \Pi_{k_{n+1}} u_{n+1}\right)=0$.

The following proposition is proven below.

Proposition 3.2. Consider four positive numbers $\nu, \delta, \tau$ and $s$. There is $s_{0}=s_{0}(\nu)>0$ such that any $\mathcal{M} \in \mathcal{M}_{n, \delta}^{\tau, \nu}$ admits a unique extension as a bounded operator from $\left(H^{s}\right)^{n}$ to $\mathcal{L}\left(H^{s}, H^{s-\tau-2}\right)$ for $s>s_{0}$. That means that the following inequality holds for any $u_{1}, \ldots, u_{n+1} \in \mathcal{D}\left(\mathbb{S}^{1} \times r \mathbb{S}^{1}\right)$

$$
\left\|\mathcal{M}\left(u_{1}, \ldots, u_{n}\right) u_{n+1}\right\|_{H^{s-\tau-2}} \leq C \prod_{j=1}^{n+1}\left\|u_{j}\right\|_{H^{s}} .
$$

Remark 3.3. In the paper [26], the sequence $\lambda_{k}$ behaves as $\sqrt{k}$ and $\lambda_{k+1}-\lambda_{k}$ as $\sqrt{k+1}-\sqrt{k}$. This separation property allows to get the stronger estimate :

$$
\left\|\mathcal{M}\left(u_{1}, \ldots, u_{n}\right) u_{n+1}\right\|_{H^{s-\tau-1}} \leq C \prod_{j=1}^{n+1}\left\|u_{j}\right\|_{H^{s}} .
$$

From now, we will call the $H^{s}$-boundedness the property which holds for any $\mathcal{M} \in \mathcal{M}_{n, \delta}^{2 s-2, \nu}$ in Proposition 3.2:

$$
\forall\left(u_{0}, u_{1}, \ldots, u_{n+1}\right) \in \mathcal{D}\left(\mathbb{S}^{1} \times r \mathbb{S}^{1}\right)^{n+2} \quad\left|\left\langle\mathcal{M}\left(u_{1}, \ldots, u_{n}\right) u_{n+1}, u_{0}\right\rangle\right| \leq C \prod_{j=0}^{n+1}\left\|u_{j}\right\|_{H^{s}}
$$

The power of $\lambda_{k_{0}}$ in the bound of the following lemma is the essential reason which allows to prove Proposition 3.2 (as explained in the introduction, that should be compared to [26, line (2.1.9)]). 
Lemma 3.4. For any integer $k_{0} \geq 1$, any real numbers $a \geq 1$ and $\omega>2$, one has

$$
\sum_{k_{n+1} \geq 1} \frac{1}{\left(\left|\lambda_{k_{n+1}}-\lambda_{k_{0}}\right|+a\right)^{\omega}} \leq \frac{C \lambda_{k_{0}}^{2}}{a^{\omega-2}}
$$

where $C>0$ is independent with respect to $\left(a, k_{0}\right)$.

Proof. We begin by separating $\mathbb{N}^{\star}$ in two subsets $S\left(\lambda_{k_{0}}\right) \sqcup T\left(\lambda_{k_{0}}\right)$ :

$$
\left.\begin{array}{l}
S\left(\lambda_{k_{0}}\right):=\left\{k_{n+1} \geq 1, \quad \lambda_{k_{n+1}} \leq 2 \lambda_{k_{0}}\right\} \\
T\left(\lambda_{k_{0}}\right):=\left\{k_{n+1} \geq 1, \quad \lambda_{k_{n+1}}>2 \lambda_{k_{0}}\right.
\end{array}\right\} .
$$

From (11) (or (14)), we obviously have $1+\max S\left(\lambda_{k_{0}}\right)=\min T\left(\lambda_{k_{0}}\right) \simeq \lambda_{k_{0}}^{2}$. So we can write

$$
\sum_{S\left(\lambda_{k_{0}}\right)} \frac{1}{\left(\left|\lambda_{k_{n+1}}-\lambda_{k_{0}}\right|+a\right)^{\omega}} \lesssim \frac{\lambda_{k_{0}}^{2}}{a^{\omega}}
$$

Note that the inequality $\lambda_{k_{n+1}}-\lambda_{k_{0}}>\frac{1}{2} \lambda_{k_{n+1}}$ holds in $T\left(\lambda_{k_{0}}\right)$, we get

$$
\sum_{T\left(\lambda_{k_{0}}\right)} \frac{1}{\left(\left|\lambda_{k_{n+1}}-\lambda_{k_{0}}\right|+a\right)^{\omega}} \lesssim \sum_{T\left(\lambda_{k_{0}}\right)} \frac{1}{\left(\sqrt{k_{n+1}}+a\right)^{\omega}} \lesssim \sum_{k_{n+1} \geq 2} \frac{1}{\left(k_{n+1}+a^{2}\right)^{\omega / 2}} \lesssim \int_{1}^{+\infty} \frac{d x}{\left(x+a^{2}\right)^{\omega / 2}}
$$

As $\omega$ is a fixed number greater than 2, the previous bound is less than or equal to $a^{-2\left(\frac{\omega}{2}-1\right)}=a^{-(\omega-2)}$, up to a multiplicative constant. We easily conclude.

Following the same lines than 26, we prove Proposition 3.2 .

Proof. Let us define

$$
\begin{aligned}
& \Omega(\delta)=\left\{\left(k_{1}, \ldots, k_{n+1}\right) \in\left(\mathbb{N}^{\star}\right)^{n+1}, \quad \delta \leq \frac{\lambda_{k_{n+1}}}{\lambda_{k_{0}}} \leq \frac{1}{\delta}, \quad \max \left(k_{1}, \ldots, k_{n}\right) \leq k_{n+1}\right\}, \\
& \Psi(\delta)=\left\{\left(k_{1}, \ldots, k_{n+1}\right) \in \Omega(\delta), \quad k_{1} \leq \cdots \leq k_{n+1}\right\} \subset \Omega(\delta) .
\end{aligned}
$$

The square of the norm $\left\|\mathcal{M}\left(u_{1}, \ldots, u_{n}\right) u_{n+1}\right\|_{H^{s-\tau-2}}$ is

$$
\begin{aligned}
\sum_{k_{0} \geq 1} \lambda_{k_{0}}^{2(s-\tau-2)}\left\|\Pi_{k_{0}}\left(\mathcal{M}\left(u_{1}, \ldots, u_{n}\right) u_{n+1}\right)\right\|_{L^{2}\left(\mathbb{S}^{1} \times r \mathbb{S}^{1}\right)}^{2} & \\
& =\sum_{k_{0} \geq 1} \lambda_{k_{0}}^{2(s-\tau-2)}\left\|\sum_{k_{1}, \ldots, k_{n+1}} \Pi_{k_{0}}\left(\mathcal{M}\left(\Pi_{k_{1}} u_{1}, \ldots, \Pi_{k_{n}} u_{n}\right) \Pi_{k_{n+1}} u_{n+1}\right)\right\|_{L^{2}\left(\mathbb{S}^{1} \times r \mathbb{S}^{1}\right)}^{2},
\end{aligned}
$$

which is less than or equal to

$$
C \sum_{k_{0} \geq 1} \lambda_{k_{0}}^{2(s-\tau-2)}\left[\sum_{\Omega(\delta)} \lambda_{k_{0}}^{\tau} \max \left(\lambda_{k_{1}}, \ldots, \lambda_{k_{n}}\right)^{\nu}\left(1+\frac{\left|\lambda_{k_{0}}-\lambda_{k_{n+1}}\right|}{\max \left(\lambda_{k_{1}}, \ldots, \lambda_{k_{n}}\right)}\right)^{-N} \prod_{j=1}^{n+1}\left\|\Pi_{k_{j}} u_{j}\right\|_{L^{2}\left(\mathbb{S}^{1} \times r \mathbb{S}^{1}\right)}\right]^{2} .
$$

The symmetry of the variables $k_{1}, \ldots, k_{n+1}$ allows to replace $\Omega(\delta)$ by $\Psi(\delta)$. Thus, it suffices to bound

$$
\begin{gathered}
\sum_{k_{0} \geq 1} \lambda_{k_{0}}^{2(s-\tau-2)}\left[\sum_{\Psi(\delta)} \lambda_{k_{n}}^{\nu} \lambda_{k_{0}}^{\tau}\left(1+\frac{\left|\lambda_{k_{0}}-\lambda_{k_{n+1}}\right|}{\lambda_{k_{n}}}\right)^{-N} \prod_{j=1}^{n+1}\left\|\Pi_{k_{j}} u_{j}\right\|_{L^{2}\left(\mathbb{S}^{1} \times r \mathbb{S}^{1}\right)}\right]^{2} \\
=\sum_{k_{0} \geq 1} \lambda_{k_{0}}^{2 s-4}\left[\sum_{\Psi(\delta)} \lambda_{k_{n}}^{\nu}\left(1+\frac{\left|\lambda_{k_{0}}-\lambda_{k_{n+1}}\right|}{\lambda_{k_{n}}}\right)^{-N} \prod_{j=1}^{n+1}\left\|\Pi_{k_{j}} u_{j}\right\|_{L^{2}\left(\mathbb{S}^{1} \times r \mathbb{S}^{1}\right)}\right]^{2} .
\end{gathered}
$$


From the Cauchy-Schwarz inequality, we bound the previous term by $C \sum_{k_{0} \geq 1} \lambda_{k_{0}}^{2 s-4} \Theta_{1} \Theta_{2}$ where $\Theta_{1}$ and $\Theta_{2}$ are defined by

$$
\begin{gathered}
\Theta_{1}:=\sum_{\Psi(\delta)} \lambda_{k_{n}}^{\nu}\left(1+\frac{\left|\lambda_{k_{0}}-\lambda_{k_{n+1}}\right|}{\lambda_{k_{n}}}\right)^{-N} \prod_{j=1}^{n}\left\|\Pi_{k_{j}} u_{j}\right\|_{L^{2}\left(\mathbb{S}^{1} \times r \mathbb{S}^{1}\right)} \\
\Theta_{2}:=\sum_{\Psi(\delta)} \lambda_{k_{n}}^{\nu}\left(1+\frac{\left|\lambda_{k_{0}}-\lambda_{k_{n+1}}\right|}{\lambda_{k_{n}}}\right)^{-N}\left\|\Pi_{k_{n+1}} u_{n+1}\right\|_{L^{2}\left(\mathbb{S}^{1} \times r \mathbb{S}^{1}\right)}^{2} \prod_{j=1}^{n}\left\|\Pi_{k_{j}} u_{j}\right\|_{L^{2}\left(\mathbb{S}^{1} \times r \mathbb{S}^{1}\right)} .
\end{gathered}
$$

For any $N=\omega>2$, Lemma 3.4 allows us to bound

$$
\begin{aligned}
\Theta_{1} & =\sum_{\Psi(\delta)} \frac{\lambda_{k_{n}}^{\nu+N}}{\left(\lambda_{k_{n}}+\left|\lambda_{k_{0}}-\lambda_{k_{n+1}}\right|\right)^{N}} \prod_{j=1}^{n}\left\|\Pi_{k_{j}} u_{j}\right\|_{L^{2}\left(\mathbb{S}^{1} \times r \mathbb{S}^{1}\right)} \\
& \leq \sum_{k_{1}, \ldots, k_{n}} \lambda_{k_{n}}^{\nu+2} \lambda_{k_{0}}^{2} \prod_{j=1}^{n}\left\|\Pi_{k_{j}} u_{j}\right\|_{L^{2}\left(\mathbb{S}^{1} \times r \mathbb{S}^{1}\right)} \\
& \leq \lambda_{k_{0}}^{2} \prod_{j=1}^{n}\left(\sum_{k_{j} \geq 1} \lambda_{k_{j}}^{\nu+2}\left\|\Pi_{k_{j}} u_{j}\right\|_{L^{2}\left(\mathbb{S}^{1} \times r \mathbb{S}^{1}\right)}\right) \\
& \leq \lambda_{k_{0}}^{2} \prod_{j=1}^{n} \sqrt{\left(\sum_{k_{j} \geq 1} \lambda_{k_{j} \geq 1}^{-2(s-\nu-2)} \lambda_{k_{j}}^{2 s}\left\|\Pi_{k_{j}} u\right\|_{L^{2}\left(\mathbb{S}^{1} \times r \mathbb{S}^{1}\right)}^{2}\right)} \\
& \lesssim \lambda_{k_{0}}^{2} \prod_{j=1}^{n}\left\|u_{j}\right\|_{H^{s}},
\end{aligned}
$$

provided that $s \gg 1$ holds (thanks to (11)). We can go on and bound $\left\|\mathcal{M}\left(u_{1}, \ldots, u_{n}\right) u_{n+1}\right\|_{H^{s-\tau-2}}^{2}$ by $\left(\sum_{k_{0} \geq 1} \lambda_{k_{0}}^{2 s-4} \times \Theta_{2} \times \lambda_{k_{0}}^{2}\right) \prod_{j=1}^{n}\left\|u_{j}\right\|_{H^{s}}$, which is nothing else than

$$
\left(\prod_{j=1}^{n}\left\|u_{j}\right\|_{H^{s}}\right) \sum_{\substack{k_{0}>1 \\ \Psi(\delta)}} \lambda_{k_{0}}^{2 s-2} \lambda_{k_{n}}^{\nu}\left(1+\frac{\left|\lambda_{k_{0}}-\lambda_{k_{n+1}}\right|}{\lambda_{k_{n}}}\right)^{-N}\left\|\Pi_{k_{n+1}} u_{n+1}\right\|_{L^{2}\left(\mathbb{S}^{1} \times r \mathbb{S}^{1}\right)}^{2} \prod_{j=1}^{n}\left\|\Pi_{k_{j}} u_{j}\right\|_{L^{2}\left(\mathbb{S}^{1} \times r \mathbb{S}^{1}\right)} .
$$

We now have to use the estimate $\lambda_{k_{n+1}} \simeq \lambda_{k_{0}}$ in the set $\Psi(\delta)$ (see $(29)$ ) to get the bound

$$
\left(\prod_{j=1}^{n}\left\|u_{j}\right\|_{H^{s}}\right) \sum_{\substack{k_{0} \geq 1 \\ \Psi(\delta)}} \frac{\lambda_{k_{n+1}}^{2 s-2} \lambda_{k_{n}}^{\nu+N}}{\left(\lambda_{k_{n}}+\left|\lambda_{k_{0}}-\lambda_{k_{n+1}}\right|\right)^{N}}\left\|\Pi_{k_{n+1}} u_{n+1}\right\|_{L^{2}\left(\mathbb{S}^{1} \times r \mathbb{S}^{1}\right)} \prod_{j=1}^{n}\left\|\Pi_{k_{j}} u_{j}\right\|_{L^{2}\left(\mathbb{S}^{1} \times r \mathbb{S}^{1}\right)} .
$$

Still using Lemma 3.4 (and inverting $k_{0}$ and $k_{n+1}$ ), we can bound

$$
\begin{aligned}
& \left(\prod_{j=1}^{n}\left\|u_{j}\right\|_{H^{s}}\right) \sum_{\Psi(\delta)} \lambda_{k_{n+1}}^{2 s} \lambda_{k_{n}}^{\nu+2}\left\|\Pi_{k_{n+1}} u_{n+1}\right\|_{L^{2}\left(\mathbb{S}^{1} \times r \mathbb{S}^{1}\right)}^{2} \prod_{j=1}^{n}\left\|\Pi_{k_{j}} u_{j}\right\|_{L^{2}\left(\mathbb{S}^{1} \times r \mathbb{S}^{1}\right)} \\
& \quad \leq C\left(\prod_{j=1}^{n}\left\|u_{j}\right\|_{H^{s}}\right)\left\|u_{n+1}\right\|_{H^{s}}^{2} \sum_{k_{1}, \ldots, k_{n}} \prod_{j=1}^{n} \lambda_{k_{j}}^{\nu+2}\left\|\Pi_{k_{j}} u_{j}\right\|_{L^{2}\left(\mathbb{S}^{1} \times r \mathbb{S}^{1}\right)},
\end{aligned}
$$

which gives the conclusion as above.

We also define nonresonant multilinear operators. 
Definition 3.5. Let $\mathcal{M}$ be an operator in $\mathcal{M}_{n, \delta}^{\tau, \nu}$ and $\ell$ be an integer in $[0, n]$. We write $\mathcal{M} \in \mathcal{M}_{n, \delta}^{\tau, \nu}[\ell]$ if for all $\left(k_{0}, \ldots, k_{n+1}\right) \in(\mathbb{N} \backslash\{0\})^{n+2}$ and $u_{1}, \ldots, u_{n+1} \in \mathcal{D}\left(\mathbb{S}^{1} \times r \mathbb{S}^{1}\right)$ we have

$$
\left\{k_{0}, \ldots, k_{\ell}\right\}=\left\{k_{\ell+1}, \ldots, k_{n+1}\right\} \Rightarrow \Pi_{k_{0}}\left(\mathcal{M}\left(\Pi_{k_{1}} u_{1}, \ldots, \Pi_{k_{n}} u_{n}\right) \Pi_{k_{n+1}} u_{n+1}\right)=0 .
$$

We also write $\mathcal{M} \in \mathcal{M}_{n, \delta}^{\tau, \nu}[\widehat{\ell}]$ if the previous implication is replaced by the following one

$$
\left\{k_{n+1}, k_{0}, \ldots, k_{\ell}\right\}=\left\{k_{\ell+1}, \ldots, k_{n}\right\} \Rightarrow \Pi_{k_{0}}\left(\mathcal{M}\left(\Pi_{k_{1}} u_{1}, \ldots, \Pi_{k_{n}} u_{n}\right) \Pi_{k_{n+1}} u_{n+1}\right)=0 .
$$

Let us define other multilinear operators.

Definition 3.6. Consider $\tau \in \mathbb{R}, \nu>0$ and an integer $\ell \in[0, n]$. A multilinear operator $\mathcal{R}: \mathcal{D}\left(\mathbb{S}^{1} \times\right.$ $\left.r \mathbb{S}^{1}\right)^{n} \rightarrow \mathcal{L}\left(\mathcal{D}\left(\mathbb{S}^{1} \times r \mathbb{S}^{1}\right), \mathcal{D}^{\prime}\left(\mathbb{S}^{1} \times r \mathbb{S}^{1}\right)\right)$ is in the class $\mathcal{R}_{n}^{\tau, \nu}[\ell]$ if the following two properties hold

i) for any $N \geq 1$ there is $C>0$ such that for any $\left(k_{0}, \ldots, k_{n+1}\right) \in(\mathbb{N} \backslash\{0\})^{n+2}, u_{1}, \ldots, u_{n+1} \in$ $\mathcal{D}\left(\mathbb{S}^{1} \times r \mathbb{S}^{1}\right)$ the $L^{2}$-norm $\left\|\Pi_{k_{0}}\left(\mathcal{R}\left(\Pi_{k_{1}} u_{1}, \ldots, \Pi_{k_{n}} u_{n}\right) \Pi_{k_{n+1}} u_{n+1}\right)\right\|_{L^{2}\left(\mathbb{S}^{1} \times r \mathbb{S}^{1}\right)}$ is less than or equal to

$$
C \lambda_{k_{0}}^{\tau} \frac{\max _{2}\left(\lambda_{k_{1}}, \ldots, \lambda_{k_{n+1}}\right)^{\nu+N}}{\left(\lambda_{k_{0}}+\cdots+\lambda_{k_{n+1}}\right)^{N}} \prod_{j=1}^{n+1}\left\|u_{j}\right\|_{L^{2}\left(\mathbb{S}^{1} \times r \mathbb{S}^{1}\right)},
$$

ii) for all $\left(k_{0}, \ldots, k_{n+1}\right) \in(\mathbb{N} \backslash\{0\})^{n+2}$ and $u_{0}, \ldots, u_{n+1} \in \mathcal{D}\left(\mathbb{S}^{1} \times r \mathbb{S}^{1}\right)$ we have

$$
\left\{k_{0}, \ldots, k_{\ell}\right\}=\left\{k_{\ell+1}, \ldots, k_{n+1}\right\} \Rightarrow \Pi_{k_{0}}\left(\mathcal{R}\left(\Pi_{k_{1}} u_{1}, \ldots, \Pi_{k_{n}} u_{n}\right) \Pi_{k_{n+1}} u_{n+1}\right)=0 .
$$

We also write $\mathcal{R} \in \mathcal{R}_{n}^{\tau, \nu}[\widehat{\ell}]$ if i) holds and if the second condition ii) is replaced by

$$
\left\{k_{n+1}, k_{0}, \ldots, k_{\ell}\right\}=\left\{k_{\ell+1}, \ldots, k_{n}\right\} \Rightarrow \Pi_{k_{0}}\left(\mathcal{R}\left(\Pi_{k_{1}} u_{1}, \ldots, \Pi_{k_{n}} u_{n}\right) \Pi_{k_{n+1}} u_{n+1}\right)=0 .
$$

As for the space $\mathcal{M}_{n, \delta}^{\tau, \nu}$, we have a $H^{s}$-boundedness property for the spaces $\mathcal{R}_{n}^{\tau, \nu}[\ell]$ and $\mathcal{R}_{n}^{\tau, \nu}[\widehat{\ell}]$.

Proposition 3.7. Consider $\nu, \tau, s>0$, there is $s_{0}=s_{0}(\nu)>0$ such that if $s$ and $3 s-\tau$ are larger than $s_{0}(\nu)$ then any operator $\mathcal{R} \in \mathcal{R}_{n}^{\tau, \nu}[\ell] \cup \mathcal{R}_{n}^{\tau, \nu}[\widehat{\ell]}$ admits a unique extension as a bounded operator from $\left(H^{s}\left(\mathbb{S}^{1} \times r \mathbb{S}^{1}\right)\right)^{n}$ to $\mathcal{L}\left(H^{s}\left(\mathbb{S}^{1} \times r \mathbb{S}^{1}\right), H^{-s}\left(\mathbb{S}^{1} \times r \mathbb{S}^{1}\right)\right)$. In other words, the following inequality holds for all $u_{1}, \ldots, u_{n+1} \in \mathcal{D}\left(\mathbb{S}^{1} \times r \mathbb{S}^{1}\right)$

$$
\left\|\mathcal{R}\left(u_{1}, \ldots, u_{n}\right) u_{n+1}\right\|_{H^{-s}\left(\mathbb{S}^{1} \times r \mathbb{S}^{1}\right)} \leq C \prod_{j=1}^{n+1}\left\|u_{j}\right\|_{H^{s}\left(\mathbb{S}^{1} \times r \mathbb{S}^{1}\right)} .
$$

Proof. The proof is the same than in [26, Proposition 2.1.5] and uses the mere asymptotic property $\lambda_{k} \simeq \sqrt{k}($ see $(11))$.

\section{Towards the regularizing effect of the beam equation}

The scope of this part is to explain the proof of Proposition 4.1 that will be used in the next parts. Remember the reduction $u:=\left(-i \partial_{t}+\sqrt{\Delta^{2}+m^{2}}\right) w$ we made to get (4). Defining $\Lambda_{m}:=\sqrt{\Delta^{2}+m^{2}}$, we have

$$
\left\|\left.\Lambda_{m}^{s / 2} u\right|_{L^{2}\left(\mathbb{S}^{1} \times r \mathbb{S}^{1}\right)} \simeq\right\| u\left\|_{H^{s}\left(\mathbb{S}^{1} \times r \mathbb{S}^{1}\right)} \simeq\right\| \partial_{t} w\left\|_{H^{s}\left(\mathbb{S}^{1} \times r \mathbb{S}^{1}\right)}+\right\| w \|_{H^{s+2}\left(\mathbb{S}^{1} \times r \mathbb{S}^{1}\right)} .
$$

Thus, if we introduce the squared Sobolev norm

$$
\Theta_{s}(u):=\frac{1}{2}\left\|\Lambda_{m}^{\frac{s}{2}} u\right\|_{L^{2}\left(\mathbb{S}^{1} \times r \mathbb{S}^{1}\right)}^{2},
$$

then Theorem 1.2 is equivalent to the proof of the following a priori estimates for all small enough number $\varepsilon>0$ :

$$
\forall t \in\left(-C \varepsilon^{-A n},+C \varepsilon^{-A n}\right) \quad \Theta_{s}(u(t)) \leq K \varepsilon^{2},
$$

for any constant $A \in\left(1, \frac{5}{4}\right)$ and some constants $K>1$ and $C>0$ (which both obviously depend on $A$ ). Such estimates will be proven in Section 5 with the aid of the following result. 
Proposition 4.1. There is a number $\delta \in(0,1)$ such that the following holds. For each integer $\ell \in[0, n]$, there are

$$
\mathcal{M}_{\ell} \in \mathcal{M}_{n, \delta}^{2 s-3, \nu}[\ell], \quad \widehat{\mathcal{M}}_{\ell} \in \mathcal{M}_{n, \delta}^{2 s-2, \nu}[\widehat{\ell}], \quad \mathcal{R}_{\ell} \in \mathcal{R}_{n}^{2 s, \nu}[\ell], \quad \widehat{\mathcal{R}}_{\ell} \in \mathcal{R}_{n}^{2 s, \nu}[\widehat{\ell}]
$$

such that

$$
\begin{aligned}
\frac{d}{d t} \Theta_{s}(u(t))= & \sum_{\ell=0}^{n} \operatorname{Re} i\left\langle\mathcal{M}_{\ell}(\underbrace{\bar{u}, \ldots, \bar{u}}_{\ell}, \underbrace{u, \ldots, u}_{n-\ell}) u, u\right\rangle \\
& +\sum_{\ell=0}^{n} \operatorname{Re} i\left\langle\mathcal{R}_{\ell}(\underbrace{\bar{u}, \ldots, \bar{u}}_{\ell}, \underbrace{u, \ldots, u}_{n-\ell}) u, u\right\rangle \\
& +\sum_{\ell=0}^{n} \operatorname{Re} i\left\langle\widehat{\mathcal{M}}_{\ell}(\underbrace{\bar{u}, \ldots, \bar{u}}_{\ell}, \underbrace{u, \ldots, u}_{n-\ell}) \bar{u}, u\right\rangle \\
& +\sum_{\ell=0}^{n} \operatorname{Re} i\left\langle\widehat{\mathcal{R}}_{\ell}(\underbrace{\bar{u}, \ldots, \bar{u}}_{\ell}, \underbrace{u, \ldots, u}_{n-\ell}) \bar{u}, u\right\rangle .
\end{aligned}
$$

The proof is quite similar to that of [26, Proposition 2.2.1] (see also a slightly modified version in [20, Proposition 4.1]) but the exponents are different. Therefore, we will write a proof which only focus on the reason why the regularizing effect of the beam equation allows for the exponent $2 s-3$ for $\mathcal{M}_{\ell}$ and skip the construction of $\widehat{M}_{\ell}, \mathcal{R}_{\ell}$ and $\widehat{R}_{\ell}$. We only recall that the construction of the previous operators rely on the multilinear estimates (15). More precisely, $\mathcal{M}_{\ell}$ and $\widehat{\mathcal{M}}_{\ell}$ are constructed thanks to (17) whereas $\mathcal{R}_{\ell}$ and $\widehat{R}_{\ell}$ are constructed thanks to $(18)$.

We stress that it is very important that the exponent $2 s-3$ is less than $2 s-2$ given by the $H^{s}$ boundedness (see (28)). As shortly explained in the introduction, this is the issue we have not overcome to deal with the Klein-Gordon equation on a Diophantine irrational torus $\mathbb{S}^{1} \times r \mathbb{S}^{1}$.

Repeating the same reasoning as in [26], there is a bounded function $b:(\mathbb{N} \backslash\{0\})^{n+1} \rightarrow \mathbb{R}$ which has support in

$$
\Gamma:=\left\{\left(k_{1}, \ldots, k_{n+1}\right) \in\left(\mathbb{N}^{\star}\right)^{n+1}, \quad \max \left(k_{1}, \ldots, k_{n}\right) \leq k_{n+1}\right\},
$$

and satisfies

$$
\begin{aligned}
w^{n+1} & =\sum_{k_{1}, \ldots, k_{n+1} \geq 1} \Pi_{k_{1}}(w) \ldots \Pi_{k_{n+1}}(w) \\
& =\sum_{\Gamma} b\left(k_{1}, \ldots, k_{n+1}\right) \prod_{j=1}^{n+1} \Pi_{k_{j}}(w) .
\end{aligned}
$$

Furthermore, the map $b$ is constant on the subset of elements $\left(k_{1}, \ldots, k_{n+1}\right) \in \Gamma$ such that $\max \left(k_{1}, \ldots, k_{n}\right)<$ $k_{n+1}$. Remember that $w$ and $u$ are related by the equality

$$
w=\Lambda_{m}^{-1}\left(\frac{u+\bar{u}}{2}\right)=\frac{1}{2} \Lambda_{m}^{-1} u+\frac{1}{2} \Lambda_{m}^{-1} \bar{u} .
$$

Putting the above expression in 30 leads us to the following formula

$$
w^{n+1}=-\sum_{\ell=0}^{n} C_{\ell}(\underbrace{\bar{u}, \ldots, \bar{u}}_{\ell}, \underbrace{u, \ldots, u}_{n-\ell}) u-\sum_{\ell=0}^{n} C_{\ell}(\underbrace{\bar{u}, \ldots, \bar{u}}_{\ell}, \underbrace{u, \ldots, u}_{n-\ell}) \bar{u},
$$

where $C_{\ell}$ is given for any $f \in \mathcal{D}\left(\mathbb{S}^{1} \times r \mathbb{S}^{1}\right)$ by

$$
\begin{aligned}
& C_{\ell}(\underbrace{\bar{u}, \ldots, \bar{u}}_{\ell}, \underbrace{u, \ldots, u}_{n-\ell}) f \\
& \quad=K_{n, \ell} \sum_{\Gamma} b\left(k_{1}, \ldots, k_{n+1}\right)\left[\prod_{j=1}^{\ell} \Pi_{k_{j}}\left(\Lambda_{m}^{-1} \bar{u}\right)\right]\left[\prod_{j=\ell+1}^{n} \Pi_{k_{j}}\left(\Lambda_{m}^{-1} u\right)\right] \Pi_{k_{n+1}}\left(\Lambda_{m}^{-1} f\right) \\
& \quad=K_{n, \ell} \sum_{\left(k_{0}, \ldots, k_{n+1}\right)} \frac{b\left(k_{1}, \ldots, k_{n+1}\right)}{\prod_{j=1}^{n+1} \sqrt{\left(\lambda_{k_{j}}^{2}-1\right)^{2}+m^{2}}} \Pi_{k_{0}}\left(\left[\prod_{j=1}^{\ell} \Pi_{k_{j}}(\bar{u})\right]\left[\prod_{j=\ell+1}^{n} \Pi_{k_{j}}(u)\right] \Pi_{k_{n+1}}(f)\right),
\end{aligned}
$$


for some constant $K_{n, \ell}$ which is a function of $n$ and $\ell$. Using $\dot{u}=i \Lambda_{m} u-i w^{n+1}$ (see (4)), we easily get

$$
\begin{aligned}
\frac{d}{d t} \Theta_{s}(u(t))= & \operatorname{Re}\left\langle\Lambda_{m}^{\frac{s}{2}} \dot{u}, \Lambda_{m}^{\frac{s}{2}} u\right\rangle=-\operatorname{Re} i\left\langle\Lambda_{m}^{\frac{s}{2}} w^{n+1}, \Lambda_{m}^{\frac{s}{2}} u\right\rangle+\overbrace{\operatorname{Re} i\left\langle\Lambda^{\frac{s+1}{2}} u, \Lambda^{\frac{s+1}{2}} u\right\rangle}^{=0} \\
= & -\operatorname{Re} i\left\langle\Lambda_{m}^{\frac{s}{2}} w^{n+1}, \Lambda_{m}^{\frac{s}{2}} u\right\rangle \\
= & \sum_{\ell=0}^{n} \operatorname{Re} i\left\langle\Lambda_{m}^{\frac{s}{2}} C_{\ell}(\underbrace{\bar{u}, \ldots, \bar{u}}_{\ell}, \underbrace{u, \ldots, u}_{n-\ell}) u, \Lambda_{m}^{\frac{s}{2}} u\right\rangle \\
& +\sum_{\ell=0}^{n} \operatorname{Re} i\left\langle\Lambda_{m}^{\frac{s}{2}} C_{\ell}(\underbrace{\bar{u}, \ldots, \bar{u}}_{\ell}, \underbrace{u, \ldots, u}_{n-\ell}) \bar{u}, \Lambda_{m}^{\frac{s}{2}} u\right\rangle .
\end{aligned}
$$

The following two lemmas will entirely prove Proposition 4.1

Lemma 4.2. There is a number $\delta \in(0,1)$ such that the following holds. For each integer $\ell \in[0, n]$, there are $\mathcal{M}_{\ell} \in \mathcal{M}_{n, \delta}^{2 s-3, \nu}[\ell]$ and $\mathcal{R}_{\ell} \in \mathcal{R}_{n}^{2 s, \nu}[\ell]$ such that

$$
\begin{aligned}
\sum_{\ell=0}^{n} \operatorname{Re} i\left\langle C_{\ell}(\underbrace{\bar{u}, \ldots, \bar{u}}_{\ell}, \underbrace{u, \ldots, u}_{n-\ell}) u, \Lambda_{m}^{s} u\right\rangle= & \sum_{\ell=0}^{n} \operatorname{Re} i\left\langle\mathcal{M}_{\ell}(\underbrace{\bar{u}, \ldots, \bar{u}}_{\ell}, \underbrace{u, \ldots, u}_{n-\ell}) u, u\right\rangle \\
& +\sum_{\ell=0}^{n} \operatorname{Re} i\left\langle\mathcal{R}_{\ell}(\underbrace{\bar{u}, \ldots, \bar{u}}_{\ell}, \underbrace{u, \ldots, u}_{n-\ell}) u, u\right\rangle .
\end{aligned}
$$

Lemma 4.3. There is a number $\delta \in(0,1)$ such that the following holds. For each integer $\ell \in[0, n]$, there are $\widehat{\mathcal{M}}_{\ell} \in \mathcal{M}_{n, \delta}^{2 s-2, \nu}[\widehat{\ell}]$ and $\widehat{\mathcal{R}}_{\ell} \in \mathcal{R}_{n}^{2 s, \nu}[\widehat{\ell}]$ such that

$$
\begin{aligned}
\sum_{\ell=0}^{n} \operatorname{Re} i\left\langle C_{\ell}(\underbrace{\bar{u}, \ldots, \bar{u}}_{\ell}, \underbrace{u, \ldots, u}_{n-\ell}) \bar{u}, \Lambda_{m}^{s} u\right\rangle= & \sum_{\ell=0}^{n} \operatorname{Re} i\left\langle\widehat{\mathcal{M}}_{\ell}(\underbrace{\bar{u}, \ldots, \bar{u}}_{\ell}, \underbrace{u, \ldots, u}_{n-\ell}) \bar{u}, u\right\rangle \\
& +\sum_{\ell=0}^{n} \operatorname{Re} i\left\langle\widehat{\mathcal{R}}_{\ell}(\underbrace{\bar{u}, \ldots, \bar{u}}_{\ell}, \underbrace{u, \ldots, u}_{n-\ell}) \bar{u}, u\right\rangle .
\end{aligned}
$$

Lemma 4.3 is similar to [26, Lemma 2.2.3]. As written above, we merely prove the part of Lemma 4.2 which involves $\mathcal{M}_{\ell}$. As in [26, page 648], we only need to consider a part of $C_{\ell}$, called below $C_{\ell, 1}$, which contains frequencies such that $\lambda_{k_{0}} \simeq \lambda_{k_{n+1}}$ and $\max \left(\lambda_{k_{1}}, \ldots, \lambda_{k_{n}}\right) \lesssim \lambda_{k_{n+1}}$. More precisely, we will consider

$C_{\ell, 1}(\underbrace{\bar{u}, \ldots, \bar{u}}_{\ell}, \underbrace{u, \ldots, u}_{n-\ell}) f:=K_{n, \ell} \sum_{\Upsilon} \frac{b\left(k_{1}, \ldots, k_{n+1}\right)}{\prod_{j=1}^{n+1} \sqrt{\left(\lambda_{k_{j}}^{2}-1\right)^{2}+m^{2}}} \Pi_{k_{0}}\left(\left[\prod_{j=1}^{\ell} \Pi_{k_{j}}(\bar{u})\right]\left[\prod_{j=\ell+1}^{n} \Pi_{k_{j}}(u)\right] \Pi_{k_{n+1}}(f)\right)$,

where $\Upsilon$ is defined, for some $\delta \in(0,1)$, by

$$
\Upsilon:=\left\{\left(k_{0}, \ldots, k_{n+1}\right) \in\left(\mathbb{N}^{\star}\right)^{n+2}, \quad \delta \leq \frac{\lambda_{k_{n+1}}}{\lambda_{k_{0}}} \leq \frac{1}{\delta}, \quad \max \left(\lambda_{k_{1}}, \ldots, \lambda_{k_{n}}\right) \leq \delta^{2} \lambda_{k_{n+1}}\right\} .
$$

Let us add that the contribution of $C_{\ell}$ which is parametrized by the complementary subset of $\Upsilon$ will indeed contribute in $\mathcal{R}_{\ell}$. Let us begin with the following equality :

$$
\operatorname{Re} i\left\langle C_{\ell, 1}(\underbrace{\bar{u}, \ldots, \bar{u}}_{\ell}, \underbrace{u, \ldots, u}_{n-\ell}) u, \Lambda_{m}^{s} u\right\rangle=-\operatorname{Re} i\left\langle C_{\ell, 1}(\underbrace{\bar{u}, \ldots, \bar{u}}_{\ell}, \underbrace{u, \ldots, u}_{n-\ell})^{\star} \Lambda_{m}^{s} u, u\right\rangle .
$$


Introducing the commutator $\left[\Lambda_{m}^{s}, C_{\ell, 1}(\underbrace{\bar{u}, \ldots, \bar{u}}_{\ell}, \underbrace{u, \ldots, u}_{n-\ell})\right]$, we get

$$
\begin{aligned}
2 \operatorname{Re} & i\left\langle\Lambda_{m}^{\frac{s}{2}} C_{\ell, 1}(\underbrace{\bar{u}, \ldots, \bar{u}}_{\ell}, \underbrace{u, \ldots, u}_{n-\ell}) u, \Lambda_{m}^{\frac{s}{2}} u\right\rangle \\
= & \operatorname{Re}\left(i\left\langle\Lambda_{m}^{s} C_{\ell, 1}(\underbrace{\bar{u}, \ldots, \bar{u}}_{\ell}, \underbrace{u, \ldots, u}_{n-\ell}) u, u\right\rangle-i\left\langle C_{\ell, 1}(\underbrace{\bar{u}, \ldots, \bar{u}}_{\ell}, \underbrace{u, \ldots, u}_{n-\ell})^{\star} \Lambda_{m}^{s} u, u\right\rangle\right) \\
= & \operatorname{Re} i\left\langle C_{\ell, 1}(\underbrace{\bar{u}, \ldots, \bar{u}}_{\ell}, \underbrace{u, \ldots, u}_{n-\ell}) \Lambda_{m}^{s} u-C_{\ell, 1}(\underbrace{\bar{u}, \ldots, \bar{u}}_{\ell}, \underbrace{u, \ldots, u}_{n-\ell})^{\star} \Lambda_{m}^{s} u, u\right\rangle \\
& +\operatorname{Re} i\left\langle\left[\Lambda_{m}^{s}, C_{\ell, 1}(\underbrace{\bar{u}, \ldots, \bar{u}}_{\ell}, \underbrace{u, \ldots, u}_{n-\ell})\right] u, u\right\rangle .
\end{aligned}
$$

Let us handle 32 . Permuting $k_{0}$ and $k_{n+1}$, we get that $C_{\ell, 1}(\underbrace{\bar{u}, \ldots, \bar{u}}_{\ell}, \underbrace{u, \ldots, u}_{n-\ell})^{\star} f$ is nothing else than

$$
\begin{gathered}
K_{n, \ell} \sum_{\Upsilon^{\star}} \frac{b\left(k_{1}, \ldots, k_{n}, k_{0}\right)}{\prod_{j=0}^{n} \sqrt{\left.\left(\lambda_{k_{j}}^{2}-1\right)^{2}+m^{2}\right)}} \Pi_{k_{0}}\left\{\Pi_{k_{n+1}}(f) \prod_{j=1}^{\ell} \Pi_{k_{j}}\left(\overline{u_{j}}\right) \prod_{j=\ell+1}^{n} \Pi_{k_{j}} u\right\}, \\
\Upsilon^{\star}:=\left\{\left(k_{0}, \ldots, k_{n+1}\right) \in\left(\mathbb{N}^{\star}\right)^{n+2}, \quad \delta \leq \frac{\lambda_{k_{n+1}}}{\lambda_{k_{0}}} \leq \frac{1}{\delta}, \quad \max \left(\lambda_{k_{1}}, \ldots, \lambda_{k_{n}}\right) \leq \delta^{2} \lambda_{k_{0}}\right\} .
\end{gathered}
$$

Let us define

$$
B\left(k_{0}, \ldots, k_{n+1}\right):=\frac{b\left(k_{1}, \ldots, k_{n+1}\right)}{\sqrt{\left.\left(\lambda_{k_{n+1}}^{2}-1\right)^{2}+m^{2}\right)}}-\frac{b\left(k_{1}, \ldots, k_{n}, k_{0}\right)}{\sqrt{\left.\left(\lambda_{k_{0}}^{2}-1\right)^{2}+m^{2}\right)}} .
$$

This definition leads us to reformulate 32 as

$$
\begin{array}{r}
K_{n, \ell} \operatorname{Re} i \sum_{\Upsilon \cap \Upsilon^{*}} B\left(k_{0}, \ldots, k_{n+1}\right) \frac{\left(\left(\lambda_{k_{n+1}}^{2}-1\right)^{2}+m^{2}\right)^{\frac{s}{2}}}{\prod_{j=1}^{n} \sqrt{\left.\left(\lambda_{k_{j}}^{2}-1\right)^{2}+m^{2}\right)}}\left\langle\prod_{j=1}^{\ell} \Pi_{k_{j}} \bar{u} \prod_{j=\ell+1}^{n+1} \Pi_{k_{j}} u, \Pi_{k_{0}} u\right\rangle \\
+K_{n, \ell} \operatorname{Re} i \sum_{\Upsilon \backslash\left(\Upsilon \cap \Upsilon^{\star}\right)} b\left(k_{1}, \ldots, k_{n+1}\right) \frac{\left(\left(\lambda_{k_{n+1}}^{2}-1\right)^{2}+m^{2}\right)^{\frac{s}{2}}}{\prod_{j=1}^{n+1} \sqrt{\left(\lambda_{k_{j}}^{2}-1\right)^{2}+m^{2}}}\left\langle\prod_{j=1}^{\ell} \Pi_{k_{j}}(\bar{u}) \prod_{j=\ell+1}^{n+1} \Pi_{k_{j}}(u), \Pi_{k_{0}}(u)\right\rangle \\
-K_{n, \ell} \operatorname{Re} i \sum_{\Upsilon^{\star} \backslash\left(\Upsilon \cap \Upsilon^{\star}\right)} b\left(k_{1}, \ldots, k_{n}, k_{0}\right) \frac{\left(\left(\lambda_{k_{n+1}}^{2}-1\right)^{2}+m^{2}\right)^{\frac{s}{2}}}{\prod_{j=0}^{n} \sqrt{\left(\lambda_{k_{j}}^{2}-1\right)^{2}+m^{2}}}\left\langle\prod_{j=1}^{\ell} \Pi_{k_{j}}(\bar{u}) \prod_{j=\ell+1}^{n+1} \Pi_{k_{j}}(u), \Pi_{k_{0}}(u)\right\rangle .
\end{array}
$$

The definition (31) of $\Upsilon$ ensures that $\max \left(k_{1}, \ldots, k_{n}\right)<\min \left(k_{0}, k_{n+1}\right)$ holds. The properties of $b$ we recalled above $\left(b\right.$ is bounded and $b\left(k_{1}, \ldots, k_{n}, k_{n+1}\right)$ takes a fixed constant if $\max \left(k_{1}, \ldots, k_{n}\right)<k_{n+1}$ holds, see (30) ) allows for the following bounds

$$
\begin{aligned}
\forall k \in \Upsilon \cap \Upsilon^{\star} \quad & \left|B\left(k_{0}, \ldots, k_{n+1}\right)\right| \lesssim\left|\frac{1}{\sqrt{\left(\lambda_{k_{0}}^{2}-1\right)^{2}+m^{2}}}-\frac{1}{\sqrt{\left(\lambda_{k_{n+1}}^{2}-1\right)^{2}+m^{2}}}\right| \lesssim \frac{\left|\lambda_{k_{0}}-\lambda_{k_{n+1}}\right|}{\lambda_{k_{n+1}}^{3}} \\
\forall k \in \Upsilon \backslash\left(\Upsilon \cap \Upsilon^{\star}\right) & \frac{\left|b\left(k_{1}, \ldots, k_{n+1}\right)\right|}{\sqrt{\left(\lambda_{k_{n+1}}^{2}-1\right)^{2}+m^{2}}} \lesssim \frac{1}{\lambda_{k_{n+1}}^{2}} \lesssim \frac{\max \left(\lambda_{k_{1}}, \ldots, \lambda_{k_{n}}\right)}{\lambda_{k_{n+1}}^{3}} \\
\forall k \in \Upsilon^{\star} \backslash\left(\Upsilon \cap \Upsilon^{\star}\right) & \frac{\left|b\left(k_{1}, \ldots, k_{n}, k_{0}\right)\right|}{\sqrt{\left(\lambda_{k_{0}}^{2}-1\right)^{2}+m^{2}}} \lesssim \frac{1}{\lambda_{k_{0}}^{2}} \lesssim \frac{\max \left(\lambda_{k_{1}}, \ldots, \lambda_{k_{n}}\right)}{\lambda_{k_{n+1}}^{3}} .
\end{aligned}
$$




$$
\begin{aligned}
\forall k \in \Upsilon \cap \Upsilon^{\star} \quad\left|B\left(k_{0}, \ldots, k_{n+1}\right)\right| & \lesssim\left|\frac{1}{\sqrt{\left(\lambda_{k_{0}}^{2}-1\right)^{2}+m^{2}}}-\frac{1}{\sqrt{\left(\lambda_{k_{n+1}}^{2}-1\right)^{2}+m^{2}}}\right| \\
& \lesssim \frac{\left|\lambda_{k_{0}}-\lambda_{k_{n+1}}\right|}{\lambda_{k_{n+1}}^{3}} \\
\forall k \in \Upsilon \backslash\left(\Upsilon \cap \Upsilon^{\star}\right) \quad \frac{\left|b\left(k_{1}, \ldots, k_{n+1}\right)\right|}{\sqrt{\left(\lambda_{k_{n+1}}^{2}-1\right)^{2}+m^{2}}} & \lesssim \frac{1}{\lambda_{k_{n+1}}^{2}} \lesssim \frac{\max \left(\lambda_{k_{1}}, \ldots, \lambda_{k_{n}}\right)}{\lambda_{k_{n+1}}^{3}} \\
\forall k \in \Upsilon^{\star} \backslash\left(\Upsilon \cap \Upsilon^{\star}\right) \quad \frac{\left|b\left(k_{1}, \ldots, k_{n}, k_{0}\right)\right|}{\sqrt{\left(\lambda_{k_{0}}^{2}-1\right)^{2}+m^{2}}} & \lesssim \frac{1}{\lambda_{k_{0}}^{2}} \lesssim \frac{\max \left(\lambda_{k_{1}}, \ldots, \lambda_{k_{n}}\right)}{\lambda_{k_{n+1}}^{3}} .
\end{aligned}
$$

Remembering [23], we define

$$
\begin{aligned}
& \bar{B}\left(k_{0}, \ldots, k_{n+1}\right):=B\left(k_{0}, \ldots, k_{n+1}\right) \mathbf{1}_{\Upsilon \cap{ }^{*}}+\frac{b\left(k_{1}, \ldots, k_{n+1}\right)}{\sqrt{\left(\lambda_{k_{n+1}}^{2}-1\right)^{2}+m^{2}}} \mathbf{1}_{\Upsilon \backslash\left(\Upsilon \cap \Upsilon^{\star}\right)}-\frac{b\left(k_{1}, \ldots, k_{n}, k_{0}\right)}{\sqrt{\left(\lambda_{k_{0}}^{2}-1\right)^{2}+m^{2}}} \mathbf{1}_{\Upsilon \backslash\left(\Upsilon \cap \Upsilon^{\star}\right)}, \\
& \mathcal{M}_{\ell, 1}\left(u_{1}, \ldots, u_{n}\right) u_{n+1}:=K_{n, \ell} \sum_{\left(\mathbb{N}^{\star}\right)^{n+2} \backslash \Omega_{n+2}(\ell)} \bar{B}\left(k_{0}, \ldots, k_{n+1}\right) \frac{\left(\left(\lambda_{k_{n+1}}^{2}-1\right)^{2}+m^{2}\right)^{\frac{s}{2}}}{\prod_{j=1}^{n} \sqrt{\left.\left(\lambda_{k_{j}}^{2}-1\right)^{2}+m^{2}\right)}} \Pi_{k_{0}}\left(\prod_{j=1}^{n+1} \Pi_{k_{j}}\left(u_{j}\right)\right) .
\end{aligned}
$$

Removing $\Omega_{n+2}(\ell)$ allows to prove, after an easy computation, that $(32)$ is equal to

$$
\operatorname{Re} i\left\langle M_{\ell, 1}(\underbrace{\bar{u}, \ldots, \bar{u}}_{\ell \text { times }}, \underbrace{u, \ldots, u}_{n-\ell \text { times }}) u, u\right\rangle .
$$

The operator $\mathcal{M}_{\ell, 1}$ is moreover nonresonant in the sense of Definition 3.5. It is also easy to prove that $\mathcal{M}_{\ell, 1}$ belongs to $\mathcal{M}_{n, \delta}^{2 s-3, \nu+2}$ for some constant $\nu>n$ with the aid of the estimates (17) and by noticing that a common upper bound of (34), (35) and (36) is $\max \left(\lambda_{k_{1}}, \ldots, \lambda_{k_{n}}\right) \lambda_{k_{n+1}}^{-3}\left(1+\left|\lambda_{k_{0}}-\lambda_{k_{n+1}}\right|\right)$ : for any $N \in \mathbb{N}^{\star}$ we can write

$$
\begin{aligned}
& \| \bar{B}\left(k_{0}, \ldots, k_{n+1}\right) \frac{\left(\left(\lambda_{k_{n+1}}^{2}-1\right)^{2}+m^{2}\right)^{\frac{s}{2}}}{\prod_{j=1}^{n} \sqrt{\left.\left(\lambda_{k_{j}}^{2}-1\right)^{2}+m^{2}\right)}} \Pi_{k_{0}}\left(\prod_{j=1}^{n+1} \Pi_{k_{j}}\left(u_{j}\right)\right) \\
& \quad \lesssim \lambda_{k_{0}}^{2 s-3}\left(1+\left|\lambda_{k_{0}}-\lambda_{k_{n+1}}\right|\right) \max \left(\lambda_{k_{1}}, \ldots, \lambda_{k_{n}}\right)^{\nu+1}\left(1+\frac{\left|\lambda_{k_{0}}-\lambda_{k_{n+1}}\right|}{\max \left(\lambda_{k_{1}}, \ldots, \lambda_{k_{n}}\right)}\right)^{-N} \prod_{j=1}^{n+1}\left\|u_{j}\right\|_{L^{2}\left(\mathbb{S}^{1} \times r \mathbb{S}^{1}\right)} \\
& \quad \lesssim \lambda_{k_{0}}^{2 s-3} \max \left(\lambda_{k_{1}}, \ldots, \lambda_{k_{n}}\right)^{\nu+2}\left(1+\frac{\left|\lambda_{k_{0}}-\lambda_{k_{n+1}}\right|}{\max \left(\lambda_{k_{1}}, \ldots, \lambda_{k_{n}}\right)}\right)^{-(N-1)} \prod_{j=1}^{n+1}\left\|u_{j}\right\|_{L^{2}\left(\mathbb{S}^{1} \times r \mathbb{S}^{1}\right)} .
\end{aligned}
$$

A similar strategy is possible to handle (33). From the definition of $C_{\ell, 1}$ (see (31) above), the commutator takes the form

$$
\begin{aligned}
& \left\langle\left[\Lambda_{m}^{s}, C_{\ell, 1}(\underbrace{\bar{u}, \ldots, \bar{u}}_{\ell}, \underbrace{u, \ldots, u}_{n-\ell})\right] u, u\right\rangle \\
& \quad=K_{n, \ell} \sum_{\Upsilon} \frac{b\left(k_{1}, \ldots, k_{n+1}\right) c_{m, s}\left(k_{0}, k_{n+1}\right)}{\prod_{j=1}^{n+1} \sqrt{\left(\lambda_{k_{j}}^{2}-1\right)^{2}+m^{2}}}\left\langle\prod_{j=1}^{\ell} \Pi_{k_{j}}(\bar{u}) \prod_{j=\ell+1}^{n+1} \Pi_{k_{j}}(u), \Pi_{k_{0}}(u)\right\rangle,
\end{aligned}
$$

with $c_{m, s}\left(k_{0}, k_{n+1}\right):=\left(\left(\lambda_{k_{0}}^{2}-1\right)^{2}+m^{2}\right)^{\frac{s}{2}}-\left(\left(\lambda_{k_{n+1}}^{2}-1\right)^{2}+m^{2}\right)^{\frac{s}{2}}$. Since $b$ is bounded, we obviously have

$$
\frac{\left|b\left(k_{1}, \ldots, k_{n+1}\right) c_{m, s}\left(k_{0}, k_{n+1}\right)\right|}{\prod_{j=1}^{n+1} \sqrt{\left(\lambda_{k_{j}}^{2}-1\right)^{2}+m^{2}}} \lesssim \frac{\left|\lambda_{k_{0}}-\lambda_{k_{n+1}}\right| \lambda_{k_{0}}^{2 s-1}}{\lambda_{k_{n+1}}^{2}} \lesssim\left|\lambda_{k_{0}}-\lambda_{k_{n+1}}\right| \lambda_{k_{0}}^{2 s-3} .
$$


We can finish as above.

The operators $\widehat{\mathcal{M}}_{\ell}$ and $\widehat{\mathcal{R}}_{\ell}$ come from Corollary 2.3 and considerations on the frequencies which respectively satisfy $\lambda_{k_{n+1}} \simeq \lambda_{k_{0}}$ and $\lambda_{k_{n+1}} \not \lambda_{k_{0}}$. Following [26, page 652], similar ideas can be used to handle $\widehat{\mathcal{M}}_{\ell}$ and we check that the following operator is convenient

$$
\widehat{\mathcal{M}}_{\ell}\left(u_{1}, \ldots, u_{n}\right) u_{n+1}:=K_{n, \ell} \sum_{\substack{\max \left(k_{1}, \ldots, k_{n}\right) \leq k_{n+1} \\ \delta \lambda_{k_{0}} \leq \lambda_{k_{n+1}} \leq \frac{1}{\delta} \lambda_{k_{0}} \\\left(k_{0}, \ldots, k_{n+1}\right) \notin \Omega_{n+2}(\widehat{\ell})}} \frac{b\left(k_{1}, \ldots, k_{n+1}\right)\left(\left(\lambda_{k_{0}}^{2}-1\right)^{2}+m^{2}\right)^{\frac{s}{2}}}{\prod_{j=1}^{n+1} \sqrt{\left(\lambda_{k_{j}}^{2}+m^{2}\right)^{2}+m^{2}}} \Pi_{k_{0}}\left(\prod_{j=1}^{n+1} \Pi_{k_{j}}\left(u_{j}\right)\right) .
$$

\section{$5 \quad$ Proof of Theorem 1.2}

The main result of the paper, namely Theorem 1.2 , will be a consequence of Proposition 5.1 and 5.2 From now, we consider the operators $\mathcal{M}_{\ell} \in \mathcal{M}_{n, \delta}^{2 s,-\nu}[\ell], \widehat{\mathcal{M}}_{\ell} \in \mathcal{M}_{n, \delta}^{2 s-2, \nu}[\widehat{\ell}], \mathcal{R}_{\ell} \in \mathcal{R}_{n}^{2 s, \nu}[\ell]$ and $\mathcal{R}_{\ell} \in \mathcal{R}_{n}^{2 s, \nu}[\ell]$ in Proposition 4.1. We choose $s$ large enough such that one can use Proposition 3.2 and Proposition 3.7 We also assume $m>0$ to be generic in the sense of Proposition 2.4.

Proposition 5.1. There are a number $\nu_{0}>0$ and three multilinear operators

$$
\underline{\widehat{\mathcal{M}}_{\ell}} \in \mathcal{M}_{n, \delta}^{2 s-3, \nu+\nu_{0}}[\widehat{\ell}], \quad \underline{\mathcal{R}_{\ell}} \in \mathcal{R}_{n}^{2 s, \nu+\nu_{0}}[\ell], \quad \underline{\widehat{\mathcal{R}}_{\ell}} \in \mathcal{R}_{n}^{2 s, \nu+\nu_{0}}[\widehat{\ell}]
$$

such that for any solution $u$ of $\dot{u}=i \Lambda_{m} u-i w^{n+1}$, one has

$$
\begin{aligned}
\frac{d}{d t}\left\langle\underline{\widehat{\mathcal{M}}_{\ell}}(\bar{u}, \ldots, \bar{u}, u, \ldots, u) \bar{u}, u\right\rangle & =\left\langle\widehat{\mathcal{M}}_{\ell}(\bar{u}, \ldots, \bar{u}, u, \ldots, u) \bar{u}, u\right\rangle+\mathcal{O}\left(\|u\|_{H^{s}}^{2 n+2}\right), \\
\frac{d}{d t}\left\langle\underline{\mathcal{R}_{\ell}}(\bar{u}, \ldots, \bar{u}, u, \ldots, u) u, u\right\rangle & =\left\langle\mathcal{R}_{\ell}(\bar{u}, \ldots, \bar{u}, u, \ldots, u) u, u\right\rangle+\mathcal{O}\left(\|u\|_{H^{s}}^{2 n+2}\right), \\
\frac{d}{d t}\left\langle\underline{\widehat{\mathcal{R}}_{\ell}}(\underbrace{\bar{u}, \ldots, \bar{u}}_{\ell}, \underbrace{u, \ldots, u}_{n-\ell}) \bar{u}, u\right\rangle & =\left\langle\widehat{\mathcal{R}}_{\ell}(\underbrace{\bar{u}, \ldots, \bar{u}}_{\ell}, \underbrace{u, \ldots, u}_{n-\ell}) \bar{u}, u\right\rangle+\mathcal{O}\left(\|u\|_{H^{s}}^{2 n+2}\right) .
\end{aligned}
$$

Proof. We merely consider $\widehat{\mathcal{M}}_{\ell}$ because the other terms are similar. Since $\widehat{\mathcal{M}}_{\ell}$ is nonresonant (see Definition 3.5), the following equality holds for any $\left(u_{0}, \ldots, u_{n+1}\right) \in \mathcal{D}\left(\mathbb{S}^{1} \times r \mathbb{S}^{1}\right)^{n+2}$ :

$$
\left\langle\widehat{\mathcal{M}}_{\ell}\left(u_{1}, \ldots, u_{n}\right) u_{n+1}, u_{0}\right\rangle=\sum_{\substack{\max \left(k_{1}, \ldots, k_{n}\right) \leq k_{n+1} \\ \delta \lambda_{k_{0}} \leq \lambda_{k_{n+1}} \leq \frac{1}{\delta} \lambda_{k_{0}} \\\left(k_{0}, \ldots, k_{n+1}\right) \notin \Omega_{n+2}(\widehat{\ell})}}\left\langle\widehat{\mathcal{M}}_{\ell}\left(\Pi_{k_{1}} u_{1}, \ldots, \Pi_{k_{n}} u_{n}\right) \Pi_{k_{n+1}} u_{n+1}, \Pi_{k_{0}} u_{0}\right\rangle .
$$

Define now the operator $\widehat{\mathcal{M}}_{\ell}$ by

$$
\left\langle\underline{\widehat{\mathcal{M}}}\left(u_{1}, \ldots, u_{n}\right) u_{n+1}, u_{0}\right\rangle=\sum_{\substack{\max \left(k_{1}, \ldots, k_{n}\right) \leq k_{n+1} \\ \delta \lambda_{k_{0}} \leq \lambda_{k_{n}+1} \leq \frac{1}{\delta} \lambda_{k_{0}} \\\left(k_{0}, \ldots, k_{n+1}\right) \notin \Omega_{n+2}(\widehat{\ell})}} \frac{\left\langle\widehat{\mathcal{M}}_{\ell}\left(\Pi_{k_{1}} u_{1}, \ldots, \Pi_{k_{n}} u_{n}\right) \Pi_{k_{n+1}} u_{n+1}, \Pi_{k_{0}} u_{0}\right\rangle}{-i \widehat{F}_{m}^{\ell}\left(\lambda_{k_{0}}, \ldots, \lambda_{k_{n+1}}\right)} .
$$


From (26), we get for any fixed integer $N \in \mathbb{N}^{\star}$ :

$$
\begin{aligned}
\left|\frac{\left\langle\widehat{\mathcal{M}}_{\ell}\left(\Pi_{k_{1}} u_{1}, \ldots, \Pi_{k_{n}} u_{n}\right) \Pi_{k_{n+1}} u_{n+1}, \Pi_{k_{0}} u_{0}\right\rangle}{i \widehat{F}_{m}^{\ell}\left(\lambda_{k_{0}}, \ldots, \lambda_{k_{n+1}}\right)}\right| \\
\quad \lesssim \frac{\lambda_{k_{0}}^{2 s-2} \max \left(\lambda_{k_{1}}, \ldots, \lambda_{k_{n}}\right)^{\nu+\nu_{0}}}{\lambda_{k_{0}}+\lambda_{k_{n+1}}}\left(1+\frac{\left|\lambda_{k_{0}}-\lambda_{k_{n+1}}\right|}{\max \left(\lambda_{k_{1}}, \ldots, \lambda_{k_{n}}\right)}\right)^{-N} \prod_{j=0}^{n+1}\left\|u_{j}\right\|_{L^{2}\left(\mathbb{S}^{1} \times r \mathbb{S}^{1}\right)} \\
\quad \lesssim \lambda_{k_{0}}^{2 s-3} \max \left(\lambda_{k_{1}}, \ldots, \lambda_{k_{n}}\right)^{\nu+\nu_{0}}\left(1+\frac{\left|\lambda_{k_{0}}-\lambda_{k_{n+1}}\right|}{\max \left(\lambda_{k_{1}}, \ldots, \lambda_{k_{n}}\right)}\right)^{-N} \prod_{j=0}^{n+1}\left\|u_{j}\right\|_{L^{2}\left(\mathbb{S}^{1} \times r \mathbb{S}^{1}\right)} \\
\Rightarrow\left\|\Pi_{k_{0}} \widehat{\mathcal{M}}_{\ell}\left(\Pi_{k_{1}} u_{1}, \ldots, \Pi_{k_{n}} u_{n}\right) \Pi_{k_{n+1}} u_{n+1}\right\|_{L^{2}\left(\mathbb{S}^{1} \times r \mathbb{S}^{1}\right)} \\
\quad \lesssim \lambda_{k_{0}}^{2 s-3} \max \left(\lambda_{k_{1}}, \ldots, \lambda_{k_{n}}\right)^{\nu+\nu_{0}}\left(1+\frac{\left|\lambda_{k_{0}}-\lambda_{k_{n+1}}\right|}{\max \left(\lambda_{k_{1}}, \ldots, \lambda_{k_{n}}\right)}\right)^{-N} \prod_{j=1}^{n+1}\left\|u_{j}\right\|_{L^{2}\left(\mathbb{S}^{1} \times r \mathbb{S}^{1}\right)} .
\end{aligned}
$$

In other words, $\widehat{\mathcal{M}}_{\ell}$ belongs to $\mathcal{M}_{n, \delta}^{2 s-3, \nu+\nu_{0}}[\widehat{\ell}]$. Using the relations $\dot{u}=-i w^{n+1}+i \Lambda_{m} u$ and $\Lambda_{m} \Pi_{k}=$ $\sqrt{\left(\lambda_{k}^{2}-1\right)^{2}+m^{2}} \Pi_{k}$, we can distribute $i \Lambda_{m} u$ and leave the terms involving $-i w^{n+1}$ in a rest called below $Q\left(w^{n+1}\right)$ :

$$
\begin{aligned}
\frac{d}{d t}\left\langle\underline{\widehat{\mathcal{M}}_{\ell}}(\underbrace{\bar{u}, \ldots, \bar{u}}_{\ell}, \underbrace{u, \ldots, u}_{n-\ell}) \bar{u}, u\right\rangle= & -i\left\langle\underline{\mathcal{M}_{\ell}}(\underbrace{\bar{u}, \ldots, \bar{u}}_{\ell}, \underbrace{u, \ldots, u}_{n-\ell}) \bar{u}, \Lambda_{m} u\right\rangle \\
& -i \sum_{k=1}^{\ell}\left\langle\underline{\widehat{\mathcal{M}}_{\ell}}(\underbrace{\bar{u}, \ldots, \bar{u}}_{k-1}, \Lambda_{m} \bar{u}, \underbrace{\bar{u}, \ldots, \bar{u}}_{\ell-k}, \underbrace{u, \ldots, u}_{n-\ell}) \bar{u}, u\right\rangle \\
& +i \sum_{k=\ell+1}^{n}\left\langle\underline{\widehat{\mathcal{M}}_{\ell}}(\underbrace{\bar{u}, \ldots, \bar{u}}_{\ell}, \underbrace{u, \ldots, u}_{k-\ell-1}, \Lambda_{m} u, \underbrace{u, \ldots, u}_{n-k}) \bar{u}, u\right\rangle \\
& -i\left\langle\widehat{\mathcal{M}}_{\ell}(\bar{u}, \ldots, \bar{u}, u, \ldots, u) \Lambda_{m} \bar{u}, u\right\rangle+Q\left(w^{n+1}\right) \\
= & \left\langle\widehat{\mathcal{M}}_{\ell}(\bar{u}, \ldots, \bar{u}, u, \ldots, u) \bar{u}, u\right\rangle+Q\left(w^{n+1}\right) .
\end{aligned}
$$

The contribution $Q\left(w^{n+1}\right)$ contains $n+2$ terms which involve $w^{n+1}$, for instance

$$
\left\langle\widehat{\mathcal{M}}_{\ell}(\underbrace{\bar{u}, \ldots, \bar{u}}_{\ell}, \underbrace{u, \ldots, u}_{n-\ell}) \bar{u}, w^{n+1}\right\rangle .
$$

Since $2 s-3$ is less than $2 s-2$, one can use Proposition 3.2 to see that $\underline{\widehat{\mathcal{M}}_{\ell}}$ is bounded from $\left(H^{s}\right)^{n}$ to $\mathcal{L}\left(H^{s}, H^{-s}\right)$. The inequalities $\left\|w^{n+1}\right\|_{H^{s}} \lesssim\|w\|_{H^{s}}^{n+1} \leq\|u\|_{H^{s}}^{n+1}$ leads us to the conclusion. The same strategy is possible to deal with the other terms. Indeed, we have to make use of the other small divisors estimates 25D, 27) and the $H^{s}$-boundedness of the families of operators $\mathcal{R}_{n}^{2 s, \nu}[\ell]$ and $\mathcal{R}_{n}^{2 s, \nu}[\widehat{\ell}]$ family (see Proposition 3.7 .

Looking at Proposition 4.1, the previous result means that one can eliminate the three terms which involve $\widehat{\mathcal{M}}_{\ell} \in \mathcal{M}_{n, \delta}^{2 s-2, \nu}[\widehat{\ell}], \mathcal{R}_{\ell} \in \mathcal{R}_{n}^{2 s, \nu}[\ell]$ and $\widehat{\mathcal{R}}_{\ell} \in \mathcal{R}_{n}^{2 s, \nu}[\widehat{\ell}]$. If one uses a similar strategy for $\mathcal{M}_{\ell} \in$ $\mathcal{M}_{n, \delta}^{2 s-3, \nu}[\ell]$, we encounter a little issue since the lost of $4+\rho$ derivatives given by $(24)$ is too bad, and thus we would obtain an operator which belongs to $\mathcal{M}_{n, \delta}^{2 s-3+4+\rho, \nu}[\ell]=\mathcal{M}_{n, \delta}^{2 s+1+\rho, \nu}[\ell]$. This is useless for us since we do not know if the previous space has the $H^{s}$-boundedness (see (28)). Therefore, a way to overcome this issue is to eliminate only a part of $\mathcal{M}_{\ell}$ (to our knowledge, such a strategy appears for the first time in [7]). Let us consider a function $\psi:(0,1) \rightarrow \mathbb{R}^{+}$such that $\lim _{\varepsilon \rightarrow 0} \psi(\varepsilon)=+\infty$ holds. We have in mind a function as $\psi(\varepsilon)=\varepsilon^{-n \theta}$ with some positive number $\theta$. Remember that the frequencies used to define $\mathcal{M}_{\ell}$ are indeed parametrized by $\Upsilon \backslash \Omega_{n+2}(\ell)$. Then, we can write $\mathcal{M}_{\ell}$ as a sum of two operators 
$\mathcal{M}_{\ell, \varepsilon}$ and $V_{\ell, \varepsilon}$ :

$$
\begin{aligned}
& \left\langle\mathcal{M}_{\ell, \varepsilon}\left(u_{1}, \ldots, u_{n}\right) u_{n+1}, u_{0}\right\rangle:=\sum_{\substack{\Upsilon \backslash \Omega_{n+2}(\ell) \\
\lambda k_{0}<\psi(\varepsilon)}}\left\langle\mathcal{M}_{\ell}\left(\Pi_{k_{1}} u_{1}, \ldots, \Pi_{k_{n}} u_{n}\right) \Pi_{k_{n+1}} u_{n+1}, \Pi_{k_{0}} u_{0}\right\rangle,
\end{aligned}
$$

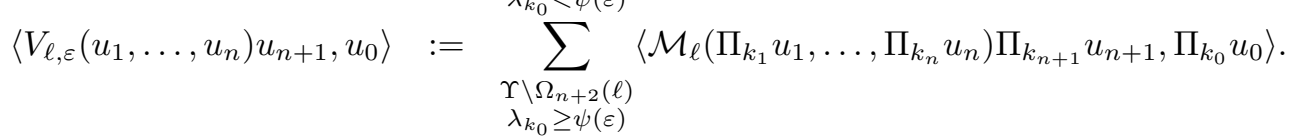

We will eliminate the $\mathcal{M}_{\ell, \varepsilon}$ part and keep the one involving $V_{\ell, \varepsilon}$. Remember that the $H^{s}$-boundedness holds for $\mathcal{M}_{n, \delta}^{2 s-2, \nu}$ and that $\mathcal{M}_{\ell}, \mathcal{M}_{\ell, \varepsilon}$ and $V_{\ell, \varepsilon}$ belong to $\mathcal{M}_{n, \delta}^{2 s-3, \nu}$. Thus, $V_{\ell, \varepsilon}$ fulfills the $H^{s}$-boundedness uniformly in $\varepsilon$ :

$$
\left|\left\langle V_{\ell, \varepsilon}\left(u_{1}, \ldots, u_{n}\right) u_{n+1}, u_{0}\right\rangle\right| \leq C \prod_{j=0}^{n+1}\left\|u_{j}\right\|_{H^{s}}
$$

Nevertheless, it is more interesting to take account the $H^{s}$-boundedness with the inequality $\lambda_{k_{0}}^{2 s-3} \leq \frac{\lambda_{k_{0}}^{2 s-2}}{\psi(\varepsilon)}$. And we get the stronger bound

$$
\left|\left\langle V_{\ell, \varepsilon}\left(u_{1}, \ldots, u_{n}\right) u_{n+1}, u_{0}\right\rangle\right| \leq \frac{C}{\psi(\varepsilon)} \prod_{j=0}^{n+1}\left\|u_{j}\right\|_{H^{s}} .
$$

The term $\mathcal{M}_{\ell, \varepsilon}$ will be eliminated by a normal form procedure.

Proposition 5.2. For any $\rho>0$ and $\varepsilon>0$, there are $\nu_{0}>0$ and $\underline{\mathcal{M}_{\ell, \varepsilon}} \in \mathcal{M}_{n, \delta}^{2 s-2, \nu+\nu_{0}}[\ell]$ such that for any solution $u$ of $\dot{u}=i \Lambda_{m} u-i w^{n+1}$, one has

$$
\frac{d}{d t}\left\langle\underline{\mathcal{M}_{\ell, \varepsilon}}(\bar{u}, \ldots, \bar{u}, u, \ldots, u) u, u\right\rangle=\left\langle\mathcal{M}_{\ell, \varepsilon}(\bar{u}, \ldots, \bar{u}, u, \ldots, u) u, u\right\rangle+\psi(\varepsilon)^{3+\rho} \mathcal{O}\left(\|u\|_{H^{s}}^{2 n+2}\right) .
$$

Proof. For any $\left(u_{0}, \ldots, u_{n+1}\right) \in \mathcal{D}\left(\mathbb{S}^{1} \times r \mathbb{S}^{1}\right)^{n+2}$, we define

$$
\left.\underline{\left\langle\mathcal{M}_{\ell, \varepsilon}\right.}\left(u_{1}, \ldots, u_{n}\right) u_{n+1}, u_{0}\right\rangle:=\sum_{\substack{\Upsilon \backslash \Omega_{n+2}(\ell) \\ \lambda k_{0}<\psi(\varepsilon)}} \frac{\left\langle\mathcal{M}_{\ell}\left(\Pi_{k_{1}} u_{1}, \ldots, \Pi_{k_{n}} u_{n}\right) \Pi_{k_{n+1}} u_{n+1}, \Pi_{k_{0}} u_{0}\right\rangle}{-i F_{m}^{\ell}\left(\lambda_{k_{0}}, \ldots, \lambda_{k_{n+1}}\right)} .
$$

From (24), the division by the small divisor gives a lost of $4+\rho$ powers of $\lambda_{k_{0}}+\lambda_{k_{n+1}}$ which is similar to $\lambda_{k_{0}}$ (because we work in $\Upsilon$ ). Consequently, we have

$$
\begin{aligned}
& \left|\frac{\left\langle\mathcal{M}_{\ell}\left(\Pi_{k_{1}} u_{1}, \ldots, \Pi_{k_{n}} u_{n}\right) \Pi_{k_{n+1}} u_{n+1}, \Pi_{k_{0}} u_{0}\right\rangle}{-i F_{m}^{\ell}\left(\lambda_{k_{0}}, \ldots, \lambda_{k_{n+1}}\right)}\right| \\
& \quad \leq C \lambda_{k_{0}}^{(2 s-3)+(4+\rho)} \max \left(\lambda_{k_{1}}, \ldots, \lambda_{k_{n}}\right)^{\nu} \frac{\max \left(\lambda_{k_{1}}, \ldots, \lambda_{k_{n}}\right)^{N+\nu_{0}}}{\left(\left|\lambda_{k_{0}}-\lambda_{k_{n+1}}\right|+\max \left(\lambda_{k_{1}}, \ldots, \lambda_{k_{n}}\right)\right)^{N}} \\
& \quad \leq C \psi(\varepsilon)^{3+\rho} \lambda_{k_{0}}^{2 s-2} \max \left(\lambda_{k_{1}}, \ldots, \lambda_{k_{n}}\right)^{\nu} \frac{\max \left(\lambda_{k_{1}}, \ldots, \lambda_{k_{n}}\right)^{N+\nu_{0}}}{\left(\left|\lambda_{k_{0}}-\lambda_{k_{n+1}}\right|+\max \left(\lambda_{k_{1}}, \ldots, \lambda_{k_{n}}\right)\right)^{N}} .
\end{aligned}
$$

Therefore, the $H^{s}$-boundedness property gives

$$
\left|\underline{\left\langle\mathcal{M}_{\ell, \varepsilon}\right.}\left(u_{1}, \ldots, u_{n}\right) u_{n+1}, u_{0}\right\rangle \mid \leq C \psi(\varepsilon)^{3+\rho} \prod_{j=0}^{n+1}\left\|u_{j}\right\|_{H^{s}}
$$

We easily conclude by computing $\frac{d}{d t}\left\langle\mathcal{M}_{\ell, \varepsilon}(\bar{u}, \ldots, \bar{u}, u, \ldots, u) u, u\right\rangle$ as in the proof of Proposition 5.1 
The proof of Theorem 1.2 is a combination of the previous results. We write it for the convenience of the reader. Let us consider $u$ a solution of $\dot{u}=i \Lambda_{m} u-i w^{n+1}$ for $s$ large enough and define

$$
\begin{aligned}
M_{s, \varepsilon}(u):= & \operatorname{Re} i \sum_{\ell=0}^{n}\left[\left\langle\underline{\mathcal{M}_{\ell, \varepsilon}}(\underbrace{\bar{u}, \ldots, \bar{u}}_{\ell}, \underbrace{u, \ldots, u}_{n-\ell}) u, u\right\rangle+\left\langle\underline{\widehat{\mathcal{M}}_{\ell}}(\underbrace{\bar{u}, \ldots, \bar{u}}_{\ell}, \underbrace{u, \ldots, u}_{n-\ell}) \bar{u}, u\right\rangle\right. \\
& \left.+\left\langle\underline{\mathcal{R}_{\ell}}(\underbrace{\bar{u}, \ldots, \bar{u}}_{\ell}, \underbrace{u, \ldots, u}_{n-\ell}) u, u\right\rangle+\left\langle\underline{\widehat{\mathcal{R}}_{\ell}}(\underbrace{\bar{u}, \ldots, \bar{u}}_{\ell}, \underbrace{u, \ldots, u}_{n-\ell}) \bar{u}, u\right\rangle\right] .
\end{aligned}
$$

The inequality (37), Proposition 4.1, Proposition 5.1 and Proposition 5.2 give us

$$
\begin{aligned}
\frac{d}{d t}\left[\Theta_{s}(u)-M_{s, \varepsilon}(u)\right] & \left.=\psi(\varepsilon)^{3+\rho} \mathcal{O}\left(\|u\|_{H^{s}}^{2 n+2}\right)+\sum_{\ell=0}^{n} \operatorname{Re} i V_{\ell, \varepsilon}(\underbrace{\bar{u}, \ldots, \bar{u}}_{\ell}, \underbrace{u, \ldots, u}_{n-\ell}) u, u\right\rangle \\
& =\psi(\varepsilon)^{3+\rho} \mathcal{O}\left(\|u\|_{H^{s}}^{2 n+2}\right)+\frac{1}{\psi(\varepsilon)} \mathcal{O}\left(\|u\|_{H^{s}}^{n+2}\right) .
\end{aligned}
$$

Remember now that the initial data $\|u(0)\|_{H^{s}}$ is of order $\varepsilon$ and that each term in $M_{s, \varepsilon}$ has the $H^{s}$ boundedness property. As we will see just below, the choice $\psi(\varepsilon)=\varepsilon^{\frac{-n}{4+\rho}}$ will be very convenient. Using (38), one sees that if $\varepsilon$ is small enough then one has

$$
\left|M_{s, \varepsilon}(u)\right| \lesssim \psi(\varepsilon)^{3+\rho} \varepsilon^{n+2}+\varepsilon^{n+2}=\varepsilon^{2+\frac{n}{4+\rho}}+\varepsilon^{n+2}=o\left(\varepsilon^{2}\right) .
$$

Integrating 39 , we get $\|u(t)\|_{H^{s}} \lesssim \varepsilon$ on an interval $[-T, T]$ such that

$$
\varepsilon^{2} \lesssim T\left(\psi(\varepsilon)^{3+\rho} \varepsilon^{n}+\frac{1}{\psi(\varepsilon)}\right) \varepsilon^{2+n}=2 T \varepsilon^{2+n+\frac{n}{4+\rho}} .
$$

We can conclude that $T \gtrsim \varepsilon^{-A n}$ holds for any fixed constant $A=1+\frac{1}{4+\rho} \in\left(1, \frac{5}{4}\right)$.

\section{Annex : proof of Proposition 2.4}

Let us consider two numbers $\wp>0, \aleph \geq 0$ and a sequence of numbers $\mu_{j} \geq 1$ such that the following two asymptotics hold as $j$ tends to infinity :

$$
\begin{aligned}
\mu_{j} & \simeq j^{\wp} \\
\mu_{j+1}-\mu_{j} & \gtrsim j^{-\aleph}
\end{aligned}
$$

For any $m>0$ and any integer $\ell \in[0, n]$, we define the following two maps which look like $F_{m}^{\ell}$ and $\widehat{F}_{m}^{\ell}$ on $[1,+\infty)^{2 n+2}($ see 22$)$ :

$$
\begin{gathered}
H_{m}^{\ell}\left(\xi_{0}, \ldots, \xi_{n+1}\right)=\sum_{j=0}^{\ell} \sqrt{\xi_{j}^{2}-1+m^{2}}-\sum_{j=\ell+1}^{n+1} \sqrt{\xi_{j}^{2}-1+m^{2}}, \\
\widehat{H}_{m}^{\ell}\left(\xi_{0}, \ldots, \xi_{n+1}\right)=\sum_{j=0}^{\ell} \sqrt{\xi_{j}^{2}-1+m^{2}}-\sum_{j=\ell+1}^{n} \sqrt{\xi_{j}^{2}-1+m^{2}}+\sqrt{\xi_{n+1}^{2}-1+m^{2}} .
\end{gathered}
$$

Indeed, the previous two maps are relevant to study the Klein-Gordon equation (2) with $\operatorname{Sp}(1-\Delta)=$ $\left\{\mu_{j}^{2}, j \geq 1\right\}$. Using the asymptotic $\mu_{j}=\sqrt{1+\left(\lambda_{j}^{2}-1\right)^{2}} \sim \lambda_{j}^{2}$ and considering $\wp=1$ and $\aleph \in(1,2)($ see Lemma 2.1, we see that Proposition 2.4 is a trivial consequence of the following result. 
Proposition 6.1. For any $\rho>0$, for almost every $m>0$ (in the sense of Lebesgue), for any $\ell \in[0, n] \cap \mathbb{N}$ there are $C>0$ and $\nu_{0}>0$ such that for any $\left(k_{0}, \ldots, k_{n+1}\right) \in(\mathbb{N} \backslash\{0\})^{n+2} \backslash \Omega_{n+2}(\ell)$ we have

$$
\begin{aligned}
& \frac{1}{\left|H_{m}^{\ell}\left(\mu_{k_{0}}, \ldots, \mu_{k_{n+1}}\right)\right|} \leq C\left(\mu_{k_{0}}+\mu_{k_{n+1}}\right)^{\max \left(\frac{\aleph}{\wp}, \frac{2}{\wp}\right)+\rho} \max \left(\mu_{k_{1}}, \ldots, \mu_{k_{n}}\right)^{\nu_{0}} \\
& \frac{1}{\left|H_{m}^{\ell}\left(\mu_{k_{0}}, \ldots, \mu_{k_{n+1}}\right)\right|} \leq C\left(\mu_{k_{0}}+\cdots+\mu_{k_{n+1}}\right)^{\nu_{0}} .
\end{aligned}
$$

In the same spirit, if $\left(k_{0}, \ldots, k_{n+1}\right) \in(\mathbb{N} \backslash\{0\})^{n+2} \backslash \Omega_{n+2}(\widehat{\ell})$ then we have

$$
\begin{gathered}
\frac{1}{\left|\widehat{H}_{m}^{\ell}\left(\mu_{k_{0}}, \ldots, \mu_{k_{n+1}}\right)\right|} \leq C \frac{\left(\mu_{k_{1}}+\cdots+\mu_{k_{n}}\right)^{\nu_{0}}}{\mu_{k_{0}}+\mu_{k_{n+1}}}, \\
\frac{1}{\left|\widehat{H}_{m}^{\ell}\left(\mu_{k_{0}}, \ldots, \mu_{k_{n+1}}\right)\right|} \leq C\left(\mu_{k_{0}}+\cdots+\mu_{k_{n+1}}\right)^{\nu_{0}} .
\end{gathered}
$$

In the case where $\aleph$ belongs to $(0,1)$, let us consider $\beta \in(0,1]$ such that $\aleph+(1-\beta) \wp$ belongs to $(0,1)$. For any $\nu_{1}>1$ there are $\nu_{0}>0$ and $C>0$ such that one can replace the right-hand side of 43 by

$$
C\left(\mu_{k_{0}}+\mu_{k_{n+1}}\right)^{\frac{1+\aleph+(1-\beta)_{\wp}}{\wp}+\rho}\left(1+\left|\mu_{k_{0}}^{\beta}-\mu_{k_{n+1}}^{\beta}\right|\right)^{\nu_{1}} \max \left(\mu_{k_{1}}, \ldots, \mu_{k_{n}}\right)^{\nu_{0}} .
$$

Here $C>0$ is independent of $\left(k_{0}, \ldots, k_{n+1}\right)$ and may depend on $\wp, \aleph, \beta, n, \ell, m, \nu_{1}$ and $\rho$.

Remark 6.2. In our paper, (47) is useless. We have written the proof because it requires an easy modification of that of (43) and may be useful for further developments. Let us add that (47) is indeed used in previous published works. For the Klein-Gordon equation on a torus $\mathbb{T}^{d}$, with $d \geq 4$, or with a quadratic potential on $\mathbb{R}^{d}$, the respective spectra $S p(-\Delta)$ and $S p\left(-\Delta+|x|^{2}\right)$ are of the form $\left\{\mu_{j}^{2}, j \geq 1\right\}$, $\mu_{j} \simeq \sqrt{j}$ and $\mu_{j+1}-\mu_{j} \gtrsim \frac{1}{\sqrt{j}}$. So one has $\wp=\aleph=\frac{1}{2}$. And thus, $\beta=1$ is convenient in [47] (see [11] and [26, line 2.3.3]).

We will follow the same idea of that of [26, Part 2.3]. Firstly, we fix the parameter $\rho>0$. Secondly, since $(0,+\infty)$ is a countable union of compact intervals, it is sufficient to prove Proposition 6.1 if $m$ belongs to a fixed compact interval $J \subset(0,+\infty)$ and if $\ell \in[0, n] \cap \mathbb{N}$ is also fixed. Note now that the following inequality holds

$$
\forall \xi \geq 1 \quad \min (1, m) \leq \frac{\sqrt{\xi^{2}-1+m^{2}}}{\xi} \leq \max (1, m) .
$$

For any $c \in \mathbb{R}$ satisfying $0<c<\min \left(1, m, \frac{1}{m}\right)$, the map $\xi \mapsto \sqrt{\xi^{2}-1+m^{2}}-c \xi$ is a positive and increasing function on $[1,+\infty)$. Hence, the compactness of $J$ allows us to choose a uniform constant $c$ on the fixed compact $J$.

\subsection{Proof of 43}

This is the big part of the proof of Proposition 6.1. Let us introduce a subset $E_{J}^{\ell}\left(k, \alpha, N_{0}\right) \subset J$, for any $\alpha>0, N_{0} \in \mathbb{N}$ and $k=\left(k_{0}, \ldots, k_{n+1}\right) \in(\mathbb{N} \backslash\{0\})^{n+2}$ by the following definition :

$$
m \in E_{J}^{\ell}\left(k, \alpha, N_{0}\right) \Leftrightarrow\left|H_{m}^{\ell}\left(\mu_{k_{0}}, \ldots, \mu_{k_{n+1}}\right)\right|<\frac{\alpha}{\left(\mu_{k_{0}}+\mu_{k_{n+1}}\right)^{\max \left(\frac{N}{6}, \frac{2}{6}\right)+\rho}\left(\mu_{k_{1}}+\cdots+\mu_{k_{n}}\right)^{N_{0}}} .
$$

Since $\mu_{k_{1}}+\cdots+\mu_{k_{n}}$ and $\max \left(\mu_{k_{1}}, \ldots, \mu_{k_{n}}\right)$ are of the same order (up to a multiplicative constant which depends on $n$ ), (43) is a consequence of the following

$$
\exists N_{0}>0 \quad \text { Leb }\left(\bigcap_{\substack{\alpha>0 \\ \alpha \in \mathbb{Q}}} \bigcup_{k \notin \Omega_{n+2}(\ell)} E_{J}^{\ell}\left(k, \alpha, N_{0}\right)\right)=0,
$$


where we denote by Leb the Lebesgue measure on $\mathbb{R}$.

We need to introduce other notations to explain the proof of $(50)$. Let us consider another family of subsets of $J$ for any number $\alpha>0, \sigma>0, N_{1} \in \mathbb{N}$ and $\widetilde{k}=\left(k_{1}, \ldots, k_{n}\right) \in\left(\mathbb{N}^{\star}\right)^{n}$ :

$$
m \in E_{J}^{\prime}\left(\widetilde{k}, \alpha^{\sigma}, N_{1}\right) \Leftrightarrow\left|\frac{\partial G_{m}^{\ell}}{\partial m}\left(\mu_{k_{1}}, \ldots, \mu_{k_{n}}\right)\right| \leq \frac{\alpha^{\sigma}}{\left(\mu_{k_{1}}+\cdots+\mu_{k_{n}}\right)^{N_{1}}},
$$

where the $\operatorname{map} G_{m}^{\ell}$ is given by

$$
\begin{aligned}
G_{m}^{\ell}:[1,+\infty)^{n} & \rightarrow \mathbb{R} \\
\left(\xi_{1}, \ldots, \xi_{p}\right) & \mapsto \sum_{j=1}^{\ell} \sqrt{m^{2}-1+\xi_{j}^{2}}-\sum_{j=\ell+1}^{n} \sqrt{m^{2}-1+\xi_{j}^{2}},
\end{aligned}
$$

and two families of subsets :

$$
\begin{aligned}
S\left(\alpha^{\sigma}, N_{1}\right) & :=\left\{k \in\left(\mathbb{N}^{\star}\right)^{n+2} \backslash \Omega_{n+2}(\ell), \quad \min \left(\mu_{k_{0}}, \mu_{k_{n+1}}\right)<\frac{1}{3 \alpha^{2 \sigma}}\left(\mu_{k_{1}}+\cdots+\mu_{k_{n}}\right)^{N_{1}}\right\}, \\
\Omega_{n}^{\prime}(\ell) & :=\left\{\left(k_{1}, \ldots, k_{n}\right) \in\left(\mathbb{N}^{\star}\right)^{n}, \quad\left\{k_{1}, \ldots, k_{\ell}\right\}=\left\{k_{\ell+1}, \ldots, k_{n}\right\}\right\} .
\end{aligned}
$$

One easily checks the following inequalities (whatever are the numbers $N_{0}, N_{1}, \sigma$ and $\alpha$ )

$$
\begin{aligned}
& \operatorname{Leb}\left(\bigcup_{k \notin \Omega_{n+2}(\ell)} E_{J}^{\ell}\left(k, \alpha, N_{0}\right)\right) \\
& \leq \operatorname{Leb}\left(\bigcup_{\substack{k \notin \Omega_{n+2}(\ell) \\
\tilde{k} \in \Omega_{n}^{\prime}(\ell)}} E_{J}^{\ell}\left(k, \alpha, N_{0}\right)\right)+\operatorname{Leb}\left(\bigcup_{\substack{k \notin \Omega_{n+2}(\ell) \\
\tilde{k} \notin \Omega_{n}^{\prime}(\ell)}} E_{J}^{\ell}\left(k, \alpha, N_{0}\right)\right) \\
& \leq \operatorname{Leb}\left(\bigcup_{\substack{k \notin \Omega_{n+2}(\ell) \\
\tilde{k} \in \Omega_{n}^{\prime}(\ell)}} E_{J}^{\ell}\left(k, \alpha, N_{0}\right)\right)+\operatorname{Leb}\left(\bigcup_{k \in S\left(\alpha^{\sigma}, N_{1}\right)} E_{J}^{\ell}\left(k, \alpha, N_{0}\right)\right) \\
& \operatorname{Leb}\left(\bigcup_{\substack{k \notin \Omega_{n+2}(\ell) \\
k \notin S\left(\alpha^{\sigma}, N_{1}\right) \\
\widetilde{k} \notin \Omega_{n}^{\prime}(\ell)}} E_{J}^{\ell}\left(k, \alpha, N_{0}\right) \cap E_{J}^{\prime \ell}\left(\widetilde{k}, \alpha^{\sigma}, N_{1}\right)^{c}\right)+\operatorname{Leb}\left(\bigcup_{\substack{k \notin \Omega_{n+2}(\ell) \\
\tilde{k} \notin \Omega_{n}^{\prime}(\ell)}} E_{J}^{\ell}\left(\widetilde{k}, \alpha^{\sigma}, N_{1}\right)\right) \text {. }
\end{aligned}
$$

The estimates of the previous four terms are given by Lemmas $6.3[6.56 .6$ and 6.7 (see below). Indeed, it will appear that there are numbers $N_{0}, N_{1}, \delta>0$ such that for any $\sigma \in\left(0, \min \left(1, \frac{N_{1}}{2 N_{0}}\right)\right)$ and $\alpha>0$ small enough we have

$$
\operatorname{Leb}\left(\bigcup_{k \notin \Omega_{n+2}(\ell)} E_{J}^{\ell}\left(k, \alpha, N_{0}\right)\right) \leq 0+C \alpha^{\delta\left(1-2 \sigma \frac{N_{0}}{N_{1}}\right)}+C \alpha^{1-\sigma}+C \alpha^{\sigma \delta},
$$

where $C>0$ is independent of $\alpha>0$. That will obviously prove 50 by making $\alpha$ tend to $0^{+}$. The following diagram explains the strategy inspired from [26, Part 2.3]. 


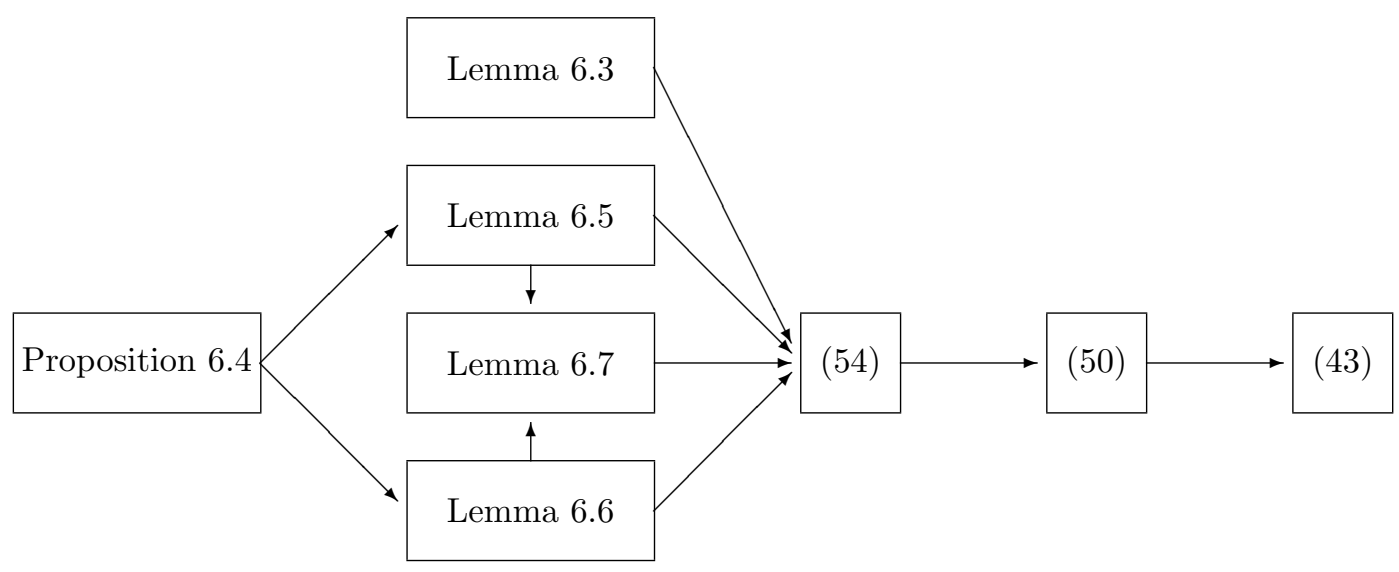

Lemma 6.3 will show why the condition (41) is useful and hence why the Diophantine assumption $r^{-2} \in \mathcal{D}($ see (3) $)$ is of interest in the study of the sequence $\mu_{j}=\sqrt{1+\left(\lambda_{j}-1\right)^{2}}$.

Lemma 6.3. There is a positive constant $c(J)$ such that if the following three conditions hold:

- $0<\alpha<c(J)$,

- $\widetilde{k}=\left(k_{1}, \ldots, k_{n}\right) \in \Omega_{n}^{\prime}(\ell)($ see $(53))$,

- $k_{0} \neq k_{n+1}$,

then $E_{J}^{\ell}\left(k, \alpha, N_{0}\right)$ is empty.

Proof. From 41] and 48, we get

$$
\begin{aligned}
\left|H_{m}^{\ell}\left(\mu_{k_{0}}, \ldots, \mu_{k_{n+1}}\right)\right| & =\left|\sqrt{\mu_{k_{0}}^{2}-1+m^{2}}-\sqrt{\mu_{k_{n+1}}^{2}-1+m^{2}}\right| \\
& =\frac{\left|\mu_{k_{0}}-\mu_{k_{n+1}}\right| \times\left|\mu_{k_{0}}+\mu_{k_{n+1}}\right|}{\left|\sqrt{\mu_{k_{n+1}}^{2}+1-m^{2}}+\sqrt{\mu_{k_{0}}^{2}+1-m^{2}}\right|} \\
& \geq \frac{c(J)}{\left(\mu_{k_{0}}+\mu_{k_{n+1}}\right)^{\frac{\aleph}{\xi}}} .
\end{aligned}
$$

From [49), the following inequality holds for any $m \in E_{J}^{\ell}\left(k, \alpha, N_{0}\right)$

$$
\begin{aligned}
\left|H_{m}^{\ell}\left(\mu_{k_{0}}, \ldots, \mu_{k_{n+1}}\right)\right| & \leq \frac{\alpha}{\left(\mu_{k_{0}}+\mu_{k_{n+1}}\right)^{\max \left(\frac{\aleph}{\varsigma}, \frac{2}{\wp}\right)+\rho}} \\
& \leq \frac{\alpha}{\left(\mu_{k_{0}}+\mu_{k_{n+1}}\right)^{\frac{\aleph}{\beta}}} .
\end{aligned}
$$

Choosing $\alpha$ small enough, we conclude that $E_{J}^{\ell}\left(k, \alpha, N_{0}\right)$ is empty.

Handling the last three terms in (54) needs to introduce several notations. Let us begin with

$$
\begin{aligned}
f^{\ell}:[0,1]^{n+3} \times J^{-1} & \rightarrow \mathbb{R} \\
\left(z, x_{0}, \ldots, x_{n+1}, y\right) & \mapsto \sum_{j=0}^{\ell} \sqrt{z^{2}+y^{2} x_{j}^{2}}-\sum_{j=\ell+1}^{n+1} \sqrt{z^{2}+y^{2} x_{j}^{2}},
\end{aligned}
$$

where we make the convention

$$
J^{-1}:=\left\{\frac{1}{m}, \quad m \in J\right\} \subset(0,+\infty) .
$$


We also need a map $g^{\ell}:[0,1]^{n+1} \times J^{-1} \rightarrow \mathbb{R}$ defined by

$$
\begin{aligned}
& z>0 \Rightarrow g^{\ell}\left(z, x_{1}, \ldots, x_{n}, y\right)=z\left[\sum_{j=1}^{\ell} \frac{z}{\sqrt{z^{2}+y^{2} x_{j}^{2}}}-\sum_{j=\ell+1}^{n} \frac{z}{\sqrt{z^{2}+y^{2} x_{j}^{2}}}\right], \\
& z=0 \Rightarrow g^{\ell}\left(z, x_{1}, \ldots, x_{n}, y\right)=0 .
\end{aligned}
$$

We also define the following map $\rho_{f}^{\ell}:[0,1]^{n+3} \rightarrow \mathbb{R}:$

$$
\begin{aligned}
& \rho_{f}^{\ell}(z, x)=z \quad \text { if } \ell \neq \frac{n}{2}, \\
& \rho_{f}^{\ell}(z, x)=z \prod_{\sigma \in \mathfrak{S}_{f, n}}\left[\sum_{j \leq \frac{n}{2}}\left(x_{\sigma(j)}^{2}-x_{j}^{2}\right)^{2}\right] \quad \text { if } \frac{n}{2} \in \mathbb{N} \quad \text { and } \ell=\frac{n}{2},
\end{aligned}
$$

where $\mathfrak{S}_{f, n}$ is the set of all bijections from $\left\{0, \ldots, \frac{n}{2}\right\}$ on $\left\{\frac{n}{2}+1, \ldots, n+1\right\}$. We define $\rho_{g}^{\ell}$ replacing in the obvious way the condition $x \in[0,1]^{n+3}$ by $x \in[0,1]^{n+1},\{0,1, \ldots, n+1\}$ by $\{1, \ldots, n\}$, and $\mathfrak{S}_{f, n}$ by $\mathfrak{S}_{g, n}$. Proposition 2.1.2 of [7] states the following result.

Proposition 6.4. There are numbers $\widetilde{N} \in \mathbb{N}, \alpha_{0}>0, \delta>0, C>0$ such that for any $\ell \in[0, n+1] \cap \mathbb{N}$, any $\alpha \in\left(0, \alpha_{0}\right), N \geq \widetilde{N}$ and $(z, x) \in[0,1]^{n+3}$ with $\rho_{f}^{\ell}(z, x)>0$, the Lebesgue measure of the subset

$$
I_{\ell}^{f}(z, x, \alpha):=\left\{y \in J^{-1}, \quad\left|f_{\ell}(z, x, y)\right|<\alpha \rho_{f}^{\ell}(z, x)^{N}\right\}
$$

is less than $C \alpha^{\delta} \rho_{f}^{\ell}(z, x)^{N \delta}$. The same is true by replacing respectively $f,[0,1]^{n+3}, \rho_{f}^{\ell}$ by $g,[0,1]^{n+1}, \rho_{g}^{\ell}$. Furthermore, there is $K=K(N) \in \mathbb{N}$ such that the set $I_{\ell}^{g}(z, x, \alpha)$ may be written as the union of at most $K$ open disjoints subintervals of $J^{-1}$.

The following lemma gives an upper bound of the Lebesgue measures of the subsets $E_{J}^{\prime \ell}\left(\widetilde{k}, \alpha^{\sigma}, N_{1}\right)$.

Lemma 6.5. Under the assumptions of Proposition 6.4 there are constants $C_{1}>0, M \in \mathbb{N}^{\star}$ such that for any $\alpha>0$ and $\sigma>0$ with $\alpha^{\sigma} \in\left(0, \alpha_{0}\right), N_{1} \in \mathbb{N}$ with $N_{1}>\max \left(M \widetilde{N}, \frac{n M}{\delta \wp}\right)$, one has

$$
\operatorname{Leb}\left[\bigcup_{\substack{k \notin \Omega_{n+2}(\ell) \\ \tilde{k} \notin \Omega_{n}^{\prime}(\ell)}} E_{J}^{\prime}\left(\widetilde{k}, \alpha^{\sigma}, N_{1}\right)\right] \leq C_{1} \alpha^{\sigma \delta} .
$$

Furthermore, each subset $E_{J}^{\prime}\left(\widetilde{k}, \alpha^{\sigma}, N_{1}\right)$ may be written as at most $K=K\left(N_{1}\right)$ disjoints subintervals of $J$.

Proof. The proof will be a consequence of Proposition 6.4. Let us introduce

$$
X:=\left\{(z, x) \in[0,1]^{n+1}, \quad \exists \widetilde{k} \in\left(\mathbb{N}^{\star}\right)^{n} \quad z=\left(\sum_{j=1}^{n} \mu_{k_{j}}\right)^{-1}, \quad \forall j \in[1, n] \cap \mathbb{N} \quad x_{j}=z \sqrt{\mu_{k_{j}}^{2}-1}\right\} .
$$

We also use the subset $X_{\ell}^{\prime n} \subset X$ of elements $(z, x)$ which correspond to an element $\widetilde{k} \in \Omega_{n}^{\prime}(\ell)$ according to the definition of $X$. Note now that one has obviously $\rho_{g}^{\ell}(z, x) \leq C(n) z$ for any $(z, x) \in X$. Let us explain why we can say more if $(z, x)$ belongs to $X \backslash X_{\ell}^{\prime n}$. Firstly, one easily checks that $\rho_{g}^{\ell}(z, x)$ is not zero. It is indeed clear if $\ell \neq \frac{n}{2}$ because $\rho_{g}^{\ell}(z, x)=z>0$ (see the definition of $X$ ). If $n$ is even and if $\ell$ equals $\frac{n}{2}$, then there are two integers $j \in\left[1, \frac{n}{2}\right]$ and $j^{\prime} \in\left[\frac{n}{2}, n\right]$ such that $k_{j} \neq k_{j^{\prime}}$. Consequently, $\mu_{k_{j}}$ and $\mu_{k_{j^{\prime}}}$ are different and the definition (57) forces $\rho_{g}^{\ell}(z, x)$ to be positive. Secondly, by using the "Diophantine condition" 41) we have

$$
x_{j}^{2}-x_{j^{\prime}}^{2}=\frac{\mu_{k_{j}}^{2}-\mu_{k_{j^{\prime}}}^{2}}{\left(\mu_{k_{1}}+\cdots+\mu_{k_{n}}\right)^{2}} \geq \frac{\left|\mu_{k_{j}}-\mu_{k_{j^{\prime}}}\right|}{\left(\mu_{k_{1}}+\cdots+\mu_{k_{n}}\right)^{2}} \gtrsim \frac{1}{\left(\mu_{k_{1}}+\cdots+\mu_{k_{n}}\right)^{2+\frac{\aleph}{\natural}}}=z^{2+\frac{\aleph}{\natural}} .
$$


In other words, there is a positive constant $M>0$ such that $z^{M} \leq \rho_{g}^{\ell}(z, x) \leq C z$ holds provided that $\widetilde{k}$ does not belong to $\Omega_{n}^{\prime}(\ell)$. We can go on as in [26, Lemma 2.3.3] by noticing

$$
\frac{\partial G_{m}^{\ell}}{\partial m}\left(\mu_{k_{1}}, \ldots, \mu_{k_{n}}\right)=\sum_{j=1}^{\ell} \frac{m}{\sqrt{m^{2}+\mu_{k_{j}}^{2}-1}}-\sum_{j=\ell+1}^{n} \frac{m}{\sqrt{m^{2}+\mu_{k_{j}}^{2}-1}}=\frac{1}{z} g_{\ell}\left(z, x_{1}, \ldots, x_{n}, y\right),
$$

where $y$ is nothing else than $\frac{1}{m}$. Hence, if $m$ belongs to $E_{J}^{\prime \ell}\left(\widetilde{k}, \alpha^{\sigma}, N_{1}\right)$ then one has

$$
\left|g^{\ell}\left(z, x_{1}, \ldots, x_{n}, y\right)\right|<\alpha^{\sigma} z^{N_{1}+1} \leq \alpha^{\sigma}\left[\rho_{g}^{\ell}(z, x)\right]^{\frac{N_{1}+1}{M}} .
$$

We now combine Proposition 6.4 and the fact that $m \in J \mapsto m^{-1} \in J^{-1}$ is a bi-Lipschitz diffeomorphism, we get

$$
\operatorname{Leb}\left[E_{J}^{\prime}\left(\widetilde{k}, \alpha^{\sigma}, N_{1}\right)\right] \leq C(J) \alpha^{\sigma \delta} z^{\left(\frac{N_{1}+1}{M}\right) \delta}=\frac{C \alpha^{\sigma \delta}}{\left(\mu_{k_{1}}+\cdots+\mu_{k_{n}}\right)^{\delta \frac{N_{1}+1}{M}}} .
$$

Summing in $\widetilde{k}=\left(k_{1}, \ldots, k_{n}\right)$ and using the asymptotic $\mu_{k} \simeq k^{\wp}$ (see 40 $)$, we get the conclusion.

To bound the Lebesgue measures of the subsets $E_{J}^{\ell}\left(k, \alpha, N_{0}\right)$, we have to make use of the sets $S\left(\alpha^{\sigma}, N_{1}\right)$ introduced in $(52)$.

Lemma 6.6. Under the assumptions of Proposition 6.4 there are constants $M, C>0$ and $\theta \in(0,1)$ such that for any $\left(N_{0}, N_{1}\right) \in\left(\mathbb{N}^{\star}\right)^{2}$ satisfying $N_{0}>\max \left(\widetilde{N} M N_{1},(n+2) \frac{M N_{1}}{\delta_{\wp}}\right)$, any $(\alpha, \sigma) \in(0,+\infty)^{2}$ with $\alpha+\alpha^{\sigma}+\alpha^{1-2 \sigma \frac{N_{0}}{N_{1}}}<\theta$ one has

$$
\operatorname{Leb}\left[\bigcup_{k \in S\left(\alpha^{\sigma}, N_{1}\right)} E_{J}^{\ell}\left(k, \alpha, N_{0}\right)\right] \leq C_{2} \alpha^{\delta\left(1-2 \sigma \frac{N_{0}}{N_{1}}\right)} .
$$

Proof.

As in Lemma 6.5 it is sufficient to get an adequate bound of Leb $\left[E_{J}^{\ell}\left(k, \alpha, N_{0}\right)\right]$ with $k \in S\left(\alpha^{\sigma}, N_{1}\right)$. We consider two cases.

First case. If $\mu_{k_{0}}+\mu_{k_{n+1}}>\frac{1}{\alpha^{2 \sigma}}\left(\mu_{k_{1}}+\cdots+\mu_{k_{n}}\right)^{N_{1}}$ then the definition 52 implies the following bound from below :

$$
\max \left(\mu_{k_{0}}, \mu_{k_{n+1}}\right) \geq \frac{2}{3 \alpha^{2 \sigma}}\left(\mu_{k_{1}}+\cdots+\mu_{k_{n}}\right)^{N_{1}} .
$$

Suppose for instance that $\max \left(\mu_{k_{0}}, \mu_{k_{n+1}}\right)=\mu_{k_{0}}$ holds. Then we have $\mu_{k_{n+1}}<\frac{1}{3} \alpha^{-2 \sigma}\left(\mu_{k_{1}}+\cdots+\mu_{k_{n}}\right)^{N_{1}}$. By remembering that $N_{1}$ and the real numbers $\mu_{j}$ are greater than or equal to 1 , we can find $c(J) \geq 1$ which depends only on the fixed compact $J$ (see the discussion after (48)) such that

$$
\begin{aligned}
H_{m}^{\ell}\left(\mu_{k_{0}}, \ldots, \mu_{k_{n+1}}\right) & \geq \sqrt{\mu_{k_{0}}^{2}-1+m^{2}}-\sqrt{\mu_{k_{n+1}}^{2}-1+m^{2}}-\sum_{j=1}^{n} \sqrt{\mu_{k_{j}}^{2}-1+m^{2}} \\
& \geq \frac{1}{c(J)}\left(\mu_{k_{0}}-\mu_{k_{n+1}}\right)-c(J) \sum_{j=1}^{n} \mu_{k_{j}} \\
& \geq\left(\frac{1}{3 c(J) \alpha^{2 \sigma}}-c(J)\right)\left(\mu_{k_{1}}+\cdots+\mu_{k_{n}}\right)^{N_{1}} .
\end{aligned}
$$

If $\alpha^{\sigma}$ is small enough, we get $H_{m}^{\ell}\left(\mu_{k_{0}}, \ldots, \mu_{k_{n+1}}\right) \geq 1$. If $\alpha<1$ moreover holds, then the set $E_{J}^{\ell}\left(k, \alpha, N_{0}\right)$ is empty because $\left|H_{m}^{\ell}\left(\mu_{k_{0}}, \ldots, \mu_{k_{n+1}}\right)\right| \leq \alpha$ holds for any $m \in E_{J}^{\ell}\left(k, \alpha, N_{0}\right)$ (see (49)).

Second case. We assume that $\mu_{k_{0}}+\mu_{k_{n+1}} \leq \alpha^{-2 \sigma}\left(\mu_{k_{1}}+\cdots+\mu_{k_{n}}\right)^{N_{1}}$ holds. Since $\alpha^{\sigma}$ belongs to $(0,1)$, we get

$$
\mu_{k_{0}}+\mu_{k_{1}}+\cdots+\mu_{k_{n}}+\mu_{k_{n+1}} \leq 2 \alpha^{-2 \sigma}\left(\mu_{k_{1}}+\cdots+\mu_{k_{n}}\right)^{N_{1}}
$$


That ensures that we have for any $m \in E_{J}^{\ell}\left(k, \alpha, N_{0}\right)$

$$
\left|H_{m}^{\ell}\left(\mu_{k_{0}}, \ldots, \mu_{k_{n+1}}\right)\right| \leq \frac{\alpha}{\left(\mu_{k_{1}}+\cdots+\mu_{k_{n}}\right)^{N_{0}}} \leq \frac{2^{\frac{N_{0}}{N_{1}}} \alpha^{1-2 \sigma \frac{N_{0}}{N_{1}}}}{\left(\mu_{k_{0}}+\cdots+\mu_{k_{n+1}}\right)^{N_{0} / N_{1}}} .
$$

We then note that

$$
f^{\ell}\left(\frac{1}{\mu_{k_{0}}+\cdots+\mu_{k_{n+1}}}, \frac{\sqrt{\mu_{k_{0}}^{2}-1}}{\mu_{k_{0}}+\cdots+\mu_{k_{n+1}}}, \ldots, \frac{\sqrt{\mu_{k_{n+1}}^{2}-1}}{\mu_{k_{0}}+\cdots+\mu_{k_{n+1}}}, \frac{1}{m}\right)
$$

is nothing else than $\frac{1}{m\left(\mu_{k_{0}}+\cdots+\mu_{k_{n+1}}\right)} H_{\ell}^{m}\left(\mu_{k_{0}}, \ldots, \mu_{k_{n+1}}\right)$ and so is less than or equal to

$$
\frac{2^{\frac{N_{0}}{N_{1}}}}{\inf (J)} \alpha^{1-2 \sigma \frac{N_{0}}{N_{1}}} \frac{1}{\left(\mu_{k_{0}}+\cdots+\mu_{k_{n+1}}\right)^{1+\frac{N_{0}}{N_{1}}}}
$$

We can conclude by using the same strategy we used in the proof of Lemma 6.5 but with $f^{\ell}$ instead of $g^{\ell}$ (defined in (56)). Let us give a sketch of the proof. Using the fact that $k$ does not belong to $\Omega_{n+2}(\ell)$ allows for the following bound

$$
\begin{aligned}
& f^{\ell}\left(\frac{1}{\mu_{k_{0}}+\cdots+\mu_{k_{n+1}}}, \frac{\sqrt{\mu_{k_{0}}^{2}-1}}{\mu_{k_{0}}+\cdots+\mu_{k_{n+1}}}, \ldots, \frac{\sqrt{\mu_{k_{n+1}}^{2}-1}}{\mu_{k_{0}}+\cdots+\mu_{k_{n}+1}}, \frac{1}{m}\right) \\
& \quad<\frac{2^{\frac{N_{0}}{N_{1}}}}{\inf (J)} \alpha^{1-2 \sigma \frac{N_{0}}{N_{1}}} \rho_{f}^{\ell}\left(\frac{1}{\mu_{k_{0}}+\cdots+\mu_{k_{n+1}}}, \frac{\sqrt{\mu_{k_{0}}^{2}-1}}{\mu_{k_{0}}+\cdots+\mu_{k_{n+1}}}, \ldots, \frac{\sqrt{\mu_{k_{n+1}}^{2}-1}}{\mu_{k_{0}}+\cdots+\mu_{k_{n+1}}}\right)^{\frac{1}{M}\left(1+\frac{N_{0}}{N_{1}}\right)},
\end{aligned}
$$

for some constant $M>0$. An application of Proposition 6.4 gives the conclusion.

Lemma 6.7. Under the assumptions of Lemma 6.5. if $N_{0}>N_{1}+\frac{n}{\wp}$ holds and if $\alpha^{\sigma}$ is small enough then one can find $C_{3}>0$ such that

$$
\operatorname{Leb}\left(\bigcup_{\substack{k \notin \Omega_{n+2}(\ell) \\ k \notin S\left(\alpha^{\sigma}, N_{1}\right) \\ \widetilde{k} \notin \Omega_{n}^{\prime}(\ell)}} E_{J}^{\ell}\left(k, \alpha, N_{0}\right) \cap E_{J}^{\prime \ell}\left(\widetilde{k}, \alpha^{\sigma}, N_{1}\right)^{c}\right) \leq C_{3} \alpha^{1-\sigma} .
$$

Proof. Consider $m \in E_{J}^{\ell}\left(k, \alpha, N_{0}\right) \cap E_{J}^{\prime}\left(\widetilde{k}, \alpha^{\sigma}, N_{1}\right)^{c}$. By introducing

$$
\Psi_{k}(m):=G_{m}^{\ell}\left(\mu_{k_{1}}, \ldots, \mu_{k_{n}}\right)+\sqrt{\mu_{k_{0}}^{2}-1+m^{2}}-\mu_{k_{0}}-\sqrt{\mu_{k_{n+1}}^{2}-1+m^{2}}+\mu_{k_{n+1}},
$$

we get

$$
\begin{aligned}
\left|\Psi_{k}(m)+\mu_{k_{0}}-\mu_{k_{n+1}}\right| & =\left|H_{m}^{\ell}\left(\mu_{k_{0}}, \ldots, \mu_{k_{n+1}}\right)\right| \\
& \leq \frac{\alpha}{\left(\mu_{k_{0}}+\mu_{k_{n+1}}\right)^{\max \left(\frac{\aleph}{6}, \frac{2}{6}\right)+\rho}\left(\mu_{k_{1}}+\cdots+\mu_{k_{n}}\right)^{N_{0}}} .
\end{aligned}
$$


Remembering [48), 51), 52 and provided that $\alpha$ is small enough, we can bound from below

$$
\begin{aligned}
\frac{\partial \Psi_{k}(m)}{\partial m} & =\frac{\partial G_{m}^{\ell}\left(\mu_{k_{1}}, \ldots, \mu_{k_{n}}\right)}{\partial m}+\frac{m}{\sqrt{\mu_{k_{0}}^{2}-1+m^{2}}}-\frac{m}{\sqrt{\mu_{k_{n+1}}^{2}-1+m^{2}}} \\
\left|\frac{\partial \Psi_{k}(m)}{\partial m}\right| & \geq \frac{\alpha^{\sigma}}{\left(\mu_{k_{1}}+\cdots+\mu_{k_{n}}\right)^{N_{1}}}-\frac{c(J)}{\mu_{k_{0}}}-\frac{c(J)}{\mu_{k_{n+1}}} \\
& \geq \frac{\alpha^{\sigma}-2 c(J) \alpha^{2 \sigma}}{\left(\mu_{k_{1}}+\cdots+\mu_{k_{n}}\right)^{N_{1}}} \\
& \geq \frac{c(J) \alpha^{\sigma}}{\left(\mu_{k_{1}}+\cdots+\mu_{k_{n}}\right)^{N_{1}}} .
\end{aligned}
$$

Thanks to Lemma 6.5. $J-E_{J}^{\prime}\left(\widetilde{k}, \alpha^{\sigma}, N_{0}\right)$ may be written as at most $K+1$ subintervals of $J$. Thus, it appears that the Lebesgue measure of the set of $m$ such that $(59)$ holds is less than

$$
\frac{2(K+1) \alpha^{1-\sigma}}{c(J)\left(\mu_{k_{0}}+\mu_{k_{n+1}}\right)^{\max \left(\frac{\aleph}{\sigma}, \frac{2}{\sigma}\right)+\rho}\left(\mu_{k_{1}}+\cdots+\mu_{k_{n}}\right)^{N_{0}-N_{1}}} .
$$

Since $\mu_{k} \simeq k^{\wp}$ holds, we finally get the conclusion by summing on $\left(k_{0}, \ldots, k_{n+1}\right) \in\left(\mathbb{N}^{\star}\right)^{n+2}$.

\subsection{Proof of 47)}

Looking at the proof of (43) in the previous section, we have to modify the definition of $E_{J}^{\ell}\left(k, \alpha, N_{0}\right)$ (see $(49))$ in the following way : let us say that $m$ belongs to $\widetilde{E}_{J}^{\ell}\left(k, \alpha, N_{0}, \nu_{1}\right)$ if and only if we have

$$
\left|H_{m}^{\ell}\left(\mu_{k_{0}}, \ldots, \mu_{k_{n+1}}\right)\right|<\frac{\alpha}{\left(\mu_{k_{0}}+\mu_{k_{n+1}}\right)^{\frac{1+\aleph+(1-\beta) \wp}{\wp}+\rho}\left(1+\left|\mu_{k_{0}}^{\beta}-\mu_{k_{n+1}}^{\beta}\right|\right)^{\nu_{1}}\left(\mu_{k_{1}}+\cdots+\mu_{k_{n}}\right)^{N_{0}}} .
$$

Lemma 6.5 remains unchanged. Lemma 6.3 has a similar proof with $\widetilde{E}_{J}^{\ell}\left(k, \alpha, N_{0}\right)$ instead of $E_{J}^{\ell}\left(k, \alpha, N_{0}\right)$. More precisely, Lemma 6.3 merely uses the bound 55 and needs that the exponent of $\mu_{k_{0}}+\mu_{k_{n+1}}$ is greater than or equal to $\frac{\pi}{\wp}$. The same remark holds for Lemma 6.6 (see the line 58 ) which holds for any $\left.m \in \widetilde{E}_{J}^{\ell}\left(k, \alpha, N_{0}\right)\right)$. Lemma 6.7 needs the convergence of the double series $\sum \frac{1}{\left(\mu_{k_{0}}+\mu_{k_{n+1}}\right)^{\frac{2}{6}+\rho}}$ (see 600). It is possible to reduce the exponent of $\mu_{k_{0}}+\mu_{k_{n+1}}$ if we modify Lemma 6.7 as follows.

Lemma 6.8. Under assumptions of Lemma 6.5, if $N_{0}>N_{1}+\frac{n}{6}$ holds and if $\alpha^{\sigma}$ is small enough then one can find $C_{3}>0$ such that

$$
\operatorname{Leb}\left(\bigcup_{\substack{k \notin \Omega_{n+2}(\ell) \\ k \notin S\left(\alpha^{\sigma}, N_{1}\right) \\ \widetilde{k} \notin \Omega_{n}^{\prime}(\ell)}} \widetilde{E}_{J}^{\ell}\left(k, \alpha, N_{0}, \nu_{1}\right) \cap E_{J}^{\prime \ell}\left(\widetilde{k}, \alpha^{\sigma}, N_{1}\right)^{c}\right) \leq C_{3} \alpha^{1-\sigma} .
$$

Proof. The same proof of that of Lemma 6.7 gives that the Lebesgue measure of $\widetilde{E}_{J}^{\ell}\left(k, \alpha, N_{0}\right) \cap$ $E_{J}^{\prime \ell}\left(\widetilde{k}, \alpha^{\sigma}, N_{1}\right)^{c}$ is less than

$$
\frac{2(K+1) \alpha^{1-\sigma}}{c(J)\left(\mu_{k_{0}}+\mu_{k_{n+1}}\right)^{\frac{1+\aleph+(1-\beta)_{\wp}}{\wp}+\rho}\left(1+\left|\mu_{k_{0}}^{\beta}-\mu_{k_{n+1}}^{\beta}\right|\right)^{\nu_{1}}\left(\mu_{k_{1}}+\cdots+\mu_{k_{n}}\right)^{N_{0}-N_{1}}} .
$$

Summing in $\left(k_{0}, \ldots, k_{n+1}\right)$, we get the condition $N_{0}-N_{1}>\frac{n}{\wp}$ and it remains to explain the convergence of the series

$$
\sum_{k_{0}, k_{n+1}} \frac{1}{\left(\mu_{k_{0}}+\mu_{k_{n+1}}\right)^{\frac{1+\aleph+(1-\beta) \wp}{\wp}+\rho}\left(1+\left|\mu_{k_{0}}^{\beta}-\mu_{k_{n+1}}^{\beta}\right|\right)^{\nu_{1}}} .
$$


It is clear that it is sufficient to prove the convergence of the above series for $\nu_{1}>1$ arbitrary near $1^{+}$. Therefore, there is no loss of generality to assume the following inequality

$$
1+\rho \wp+\left(1-\nu_{1}\right)[\aleph+(1-\beta) \wp]>1 .
$$

The subseries of (61) on the subset $\left\{k_{0}=k_{n+1}\right\}$ is clearly convergent because of the asympotic $\mu_{k} \simeq k^{\wp}$ and the inequality $1+\aleph+(1-\beta)_{\wp} \geq 1$. Using [40), [41) and the fact that $\beta$ belongs to $(0,1]$, we easily get

$$
\left|\mu_{k_{0}}^{\beta}-\mu_{k_{n+1}}^{\beta}\right| \geq \beta \min \left(\mu_{k_{0}}^{\beta-1}, \mu_{k_{n+1}}^{\beta-1}\right)\left|\mu_{k_{0}}-\mu_{k_{n+1}}\right| \gtrsim \frac{\left|k_{0}-k_{n+1}\right|}{\left(k_{0}+k_{n+1}\right)^{\aleph+(1-\beta)_{\wp}}} .
$$

Hence, the sum of the double subseries on the subset $\left\{k_{0} \neq k_{n+1}\right\}$ is less than or equal to

$$
C \sum_{k_{0} \neq k_{n+1}} \frac{1}{\left(k_{0}+k_{n+1}\right)^{1+\rho \wp+\left(1-\nu_{1}\right)[\aleph+(1-\beta) \wp]}\left|k_{0}-k_{n+1}\right|^{\nu_{1}}},
$$

which is also convergent thanks to 62].

\subsection{Proof of 44 and 46}

We only consider the inequality (44). The other one is similar. We have indeed to work as in the second case of Lemma 6.6 and bound the Lebesgue measure of the following subset for any $\alpha>0, N_{2} \in \mathbb{N}^{*}$, $\ell \in[0, n] \cap \mathbb{N}$ and $k \in(\mathbb{N} \backslash\{0\})^{n+2} \backslash \Omega_{n+2}(\ell):$

$$
\left\{m \in J, \quad \mid H_{\ell}^{m}\left(\mu_{k_{0}}, \ldots, \mu_{k_{n+1}}\right) \leq \alpha\left(\mu_{k_{0}}+\cdots+\mu_{k_{n+1}}\right)^{-N_{2}}\right\} .
$$

\subsection{Proof of 45}

We consider two subcases.

First case. If $\mu_{k_{0}}+\mu_{k_{n+1}} \lesssim \mu_{k_{1}}+\cdots+\mu_{k_{n}}$ holds we use 460 and

$$
\left(\mu_{k_{0}}+\cdots+\mu_{k_{n+1}}\right)^{\nu_{0}} \lesssim\left(\mu_{k_{1}}+\cdots+\mu_{k_{n}}\right)^{\nu_{0}}=\frac{\left(\mu_{k_{1}}+\cdots+\mu_{k_{n}}\right)^{\nu_{0}+1}}{\mu_{k_{1}}+\cdots+\mu_{k_{n}}} \lesssim \frac{\left(\mu_{k_{1}}+\cdots+\mu_{k_{n}}\right)^{\nu_{0}+1}}{\mu_{k_{0}}+\mu_{k_{n+1}}} .
$$

Second case. If $\mu_{k_{1}}+\cdots+\mu_{k_{n}} \lesssim \mu_{k_{0}}+\mu_{k_{n+1}}$ holds then one has $\widehat{H}_{m}^{\ell}\left(\mu_{k_{0}}, \ldots, \mu_{k_{n+1}}\right) \gtrsim 1$ (see 42 and the quite similar argument in (57)). The inequality (45) is finally obvious.

ACKnowledgments. The author would like to thank Kristell Dréau for valuable discussions about beam equations.

\section{References}

[1] Dario Bambusi. Birkhoff normal form for some nonlinear PDEs. Comm. Math. Physics, 234:253-285, 2003.

[2] Dario Bambusi. A Birkhoff normal form theorem for some semilinear pdes. In Hamiltonian Dynamical Systems and Applications, pages 213-247. Springer, 2007.

[3] Dario Bambusi, Jean-Marc Delort, Benoit Grébert, and Jeremie Szeftel. Almost global existence for Hamiltonian semilinear Klein-Gordon equations with small Cauchy data on Zoll manifolds. Comm. Pure Appl. Math, 60 no 11:pages 1665-1690, 2007.

[4] Dario Bambusi and Benoît Grébert. Birkhoff normal form for PDEs with tame modulus. Duke Math. J., 135:507-567, 2006. 
[5] Dario Bambusi and Yannick Sire. Almost global existence for a fractional Schrödinger equation on spheres and tori. Dynamics of PDE, 10(2):171-176, 2013.

[6] Jean Bourgain. Construction of approximative and almost periodic solutions of perturbed linear Schrödinger and wave equations. Geometric and Functional Analysis, 6(2):201-230, 1996.

[7] Jean-Marc Delort. On long time existence for small solutions of semi-linear Klein-Gordon equations on the torus. Journal d'Analyse Mathématique, 107(1):161-194, 2009.

[8] Jean-Marc Delort. A quasi-linear Birkhoff normal forms method. Application to the quasi-linear Klein-Gordon equation on S1, volume 341 of Astérisque. Société Mathématique de France, 2012.

[9] Jean-Marc Delort and Jeremie Szeftel. Long-time existence for small data nonlinear Klein-Gordon equations on tori and spheres. Internat. Math. Res. Notices, 37:1897-1966, 2004.

[10] Jean-Marc Delort and Jeremie Szeftel. Long-Time existence for semi-linear Klein-Gordon equations with small Cauchy data on Zoll manifolds. Amer. J. Math, 128:1187-1218, 2006.

[11] Daoyuan Fang and Qidi Zhang. Long-time existence for semi-linear Klein-Gordon equations on tori. Journal of Differential Equations, 249(1):151-179, 2010.

[12] Erwan Faou. Geometric numerical integration and Schrödinger equations. European Mathematical Society, 2012.

[13] Erwan Faou and Benoît Grebert. Quasi-invariant modified Sobolev norms for semi linear reversible pdes. Nonlinearity, 23:pages 429-443, 2010.

[14] Erwan Faou and Benoit Grébert. A Nekhoroshev type theorem for the nonlinear Schrodinger equation on the torus. Analysis and PDE, 6:1243-1262, 2013.

[15] Jiansheng Geng and Jiangong You. A KAM theorem for Hamiltonian partial differential equations in higher dimensional spaces. Communications in mathematical physics, 262(2):343-372, 2006.

[16] Jiansheng Geng and Jiangong You. KAM tori for higher dimensional beam equations with constant potentials. Nonlinearity, 19(10):2405-2423, 2006.

[17] Benoit Grébert. Birkhoff normal form and Hamiltonian PDEs. In Partial differential equations and applications, volume 15 of Sémin. Congr., pages 1-46. Soc. Math. France, Paris, 2007.

[18] Marc Hindry and Joseph H. Silverman. Diophantine geometry: an introduction, volume 201. Springer, 2000.

[19] Rafik Imekraz. Existence en temps grand pour des équations de Klein-Gordon à petite donnée initiale sur une structure de Toeplitz. J. Funct. Anal., 264(6):1480-1524, 2013.

[20] Rafik Imekraz. Normal form for semi-linear Klein-Gordon equations with superquadratic oscillators. Monatsh.Math., 179(4):535-575, 2016.

[21] Huawei Niu and Jiansheng Geng. Almost periodic solutions for a class of higher-dimensional beam equations. Nonlinearity, 20(11):2499-2518, 2007.

[22] Benoit Pausader. Scattering and the Levandosky-Strauss conjecture for fourth-order nonlinear wave equations. Journal of Differential Equations, 241(2):237-278, 2007.

[23] Benoit Pausader. Scattering for the beam equation in low dimensions. Indiana Univ. Math, 593:791-822, 2010.

[24] Klaus Friedrich Roth. Rational approximations to algebraic numbers. Mathematika, 2:1-20, 61955.

[25] Wolfgang M. Schmidt. Diophantine approximations and Diophantine equations, volume 1467 of Lecture Notes in Mathematics. Springer-Verlag, Berlin, 1991.

[26] Qidi Zhang. Long-Time Existence for Semi-Linear Klein-Gordon Equations with Quadratic Potential. Communications in Partial Differential Equations, 35(4):630-668, 2010. 